\title{
Strongly first order phase transition in the singlet fermionic dark matter model after LUX
}

\section{Tai Li and Yu-Feng Zhou}

State Key Laboratory of Theoretical Physics, Kavli Institute for Theoretical Physics China, Institute of Theoretical Physics, Chinese Academy of Sciences, Beijing, 100190, P.R. China

E-mail: litai@itp.ac.cn, yfzhou@itp.ac.cn

ABSTRACT: We investigate an extension of the standard model (SM) with a singlet fermionic dark matter (DM) particle which interacts with the SM sector through a real singlet scalar. The presence of a new scalar provides the possibility of generating a strongly first order phase transition needed for electroweak baryogenesis. Taking into account the latest Higgs search results at the LHC and the upper limits from the DM direct detection experiments especially that from the LUX experiment, and combining the constraints from the LEP experiment and the electroweak precision test, we explore the parameter space of this model which can lead to the strongly first order phase transition. Both the tree- and loop-level barriers are included in the calculations. We find that the allowed mass of the second Higgs particle is in the range $\sim 30-350 \mathrm{GeV}$. The allowed mixing angle $\alpha$ between the SM-like Higgs particle and the second Higgs particle is constrained to $\alpha \lesssim 28^{\circ}$. The DM particle mass is predicted to be in the range $\sim 15-350 \mathrm{GeV}$. The future XENON1T experiment can rule out a significant proportion of the parameter space of this model. The constraint can be relaxed only when the mass of the SM-like Higgs particle is degenerate with that of the second Higgs particle, or the mixing angle is small enough.

Keywords: Higgs Physics, Beyond Standard Model

ArXiv EPRINT: 1402.3087v4 


\section{Contents}

1 Introduction 1

2 Singlet fermionic dark matter model 4

3 Effective potential and EWPhT 5

4 Parameter space for EWPhT $\quad 8$

5 The effect of the sphaleron magnetic moment 11

$6 \quad$ DM thermal relic density 12

$\begin{array}{lll}7 & \text { Direct detection of DM } & 14\end{array}$

8 Higgs signal strength at the LHC $\quad 15$

$\begin{array}{lll}9 & \text { LEP constraint and the electroweak precision test } & 17\end{array}$

$\begin{array}{ll}10 \text { Combined results } & 18\end{array}$

11 Conclusion $\quad 20$

$\begin{array}{ll}\text { A Renormalization of the Higgs potential } & 21\end{array}$

B Cross sections for DM annihilation $\quad 22$

$\begin{array}{ll}\text { C Sphaleron solution with magnetic moment } & 25\end{array}$

\section{Introduction}

The possibility of baryogenesis through electroweak phase transition (EWPhT) has been studied extensively (for reviews see e.g. refs. [1-4]). If the EWPhT is strongly first order, it can fulfill the condition of departure from thermal equilibrium which is one of the three conditions necessary for the generation of baryon number asymmetry in the Universe $[5,6]$. In order to avoid the washout of the baryon number asymmetry, the baryon number violating interactions induced by electroweak sphalerons must be suppressed at the temperature when the bubbles enveloping the broken phase start to nucleate [7]. A commonly adopted assumption is that the sphaleronic interactions are suppressed immediately after the EW$\mathrm{PhT}$, which leads to a requirement that $\varphi_{c}$ the vacuum expectation value (VEV) of the Higgs field in the broken phase is larger than the critical temperature, namely $[8,9]$

$$
\frac{\varphi_{c}}{T_{c}} \gtrsim \mathcal{E}
$$


where $\mathcal{E} \approx 1$ is a constant. In the standard model (SM), the condition in eq. (1.1) is satisfied only when the Higgs boson is very light, i.e., $m_{h} \lesssim 30 \mathrm{GeV}$ for $\mathcal{E}=1$ [10-14], which is ruled out by the current experiments, especially after the discovery of a $125 \mathrm{GeV}$ Higgs boson at the LHC $[15,16]$. Thus new physics beyond the SM must be introduced for a successful electroweak baryogenesis.

Another clear indication of new physics is the existence of dark matter (DM), which has been well established by astrophysical and cosmological observations as well as N-body simulations. According to the latest analysis reported by the Planck Collaboration, the measured energy density of DM in the Universe is [17]

$$
\Omega h^{2}=0.1187 \pm 0.0017
$$

Although the SM has been very successful in phenomenology, it can provide neither a strongly first order EWPhT for baryogenesis nor a valid candidate of DM.

One of the simplest models with DM candidates is the extension of the SM with a gauge singlet scalar field [18-26]. The stability of the scalar can be protected by an ad hoc $Z_{2}$ symmetry. The $Z_{2}$ symmetry may be a residual symmetry from a global or local U(1). In the extension of the left-right symmetric models with a gauge singlet scalar, the $Z_{2}$ symmetry may originate from the parity and CP symmetries [27-31]. However, if EWPhT is also required, it was shown that the singlet scalar could contribute only up to $3 \%$ of the $\mathrm{DM}$ energy density $[32,33]$. In the inert doublet model, an additional $\mathrm{SU}(2)$ doublet is added to the SM. This model can provide a valid DM candidate and also trigger strongly first order EWPhT, due to the contributions from other charged and neutral scalars in the additional doublet [34]. When taking into account the data of the LHC and DM direct detection experiments, the parameter space of this model is highly constrained $[35,36]$.

The DM particle can also be a gauge singlet fermion which interacts with the SM sector through a gauge singlet real scalar. The phenomenology of this type of DM model has been explored in refs. [37-41]. Light subGeV-scale singlet scalars exchanged by the fermionic DM particles can serve as a force-carrier in the mechanism of the Sommerfeld enhancement which has been considered to explain the large boost factors suggested by the data of various DM indirect detection experiments (see e.g. refs. [42-51]), such as PAMELA [52], Fermi-LAT [53, 54] and AMS-02 [55] (for a recent analysis see e.g. [56]).

It is of interest to investigate whether the strongly first order EWPhT can also be realized in the singlet fermionic DM model. This question was addressed in ref. [57] in which the discussion was limited to the case of tree-level barrier only. However, without the $Z_{2}$ symmetry, the strongly first order EWPhT can be achieved from the singlet scalar contributions via both tree- and loop-level effects due to the linear and cubic terms in the singlet scalar and Higgs potential, which is similar with the case of the SM plus a gauge singlet real scalar [58-65]. In this work, we aim at an extensive and up-to-date analysis of the EWPhT in this model. In comparison with the previous analysis, we make the following improvements:

- We go beyond the tree-level analysis by including the loop-level barrier induced from the thermal corrections to the effective potential. We show that when taking into 
account both the tree- and loop-level barriers the allowed parameter space is significantly enlarged. For instance, the upper limit on the mass of the second Higgs particle is about $100 \mathrm{GeV}$ higher at $\sin \alpha=0.001$. At the same time the critical temperature after including the cubic terms from one-loop corrections is about $10 \%$ higher. We show that in this case the allowed mass of the second Higgs particle can reach $\sim 600 \mathrm{GeV}$.

- We adopt an improved analytical approximation of the finite temperature effective potential which well matches both the usual high- and low-temperature approximations. This approximation makes our analysis valid for large values of $\varphi_{c} / T_{c}$, which is of crucial importance as the value of $\varphi_{c} / T_{c}$ can reach up to 10 in this model.

- We consider the contribution from the sphaleron magnetic moment to the sphaleron energy. We find that in this model the contribution from the sphaleron magnetic moment is weakened compared with the case of the SM, due to the extra scalar field. The sphaleron magnetic moment energy can lead to a difference between the values of $\varphi_{c} / T_{c}$ and $E_{\mathrm{sph}}\left(T_{c}\right) / 35 T_{c}$ within $10 \%$.

- We include the latest upper limits on DM-neucleon scattering cross section from the LUX experiment [66] which is about one order of magnitude stronger than the previous one reported by XENON100 [67]. As a consequence the mixing angle between the SM-like and the second Higgs particles is stringently constrained.

- We focus on the constraints on the phenomenologically interesting physical parameters such as the mass of the second Higgs particle, the mixing angle and the DM particle mass. A numerical scan of the parameter space of this model is performed using a Markov Chain Monte Carlo (MCMC) approach. Taking into account the latest data from the LHC and the LUX experiments, and combining the constrains from the LEP experiment and the electroweak precision test, we find that the mass of the second Higgs particle is in the range $\sim 30-350 \mathrm{GeV}$ and the mixing angle is constrained to $\alpha \lesssim 28^{\circ}$. We also find that the DM particle mass is predicted to be in the range $\sim 15-350 \mathrm{GeV}$.

This paper is organized as follows. We first give a brief overview of the singlet fermionic DM model in section 2. In section 3, we discuss the effective potential at finite temperature at the tree- and loop-level. A numerical analysis of parameter space is performed and the allowed parameter space is given in section 4 . In section 5 we discuss the correction of the sphaleron energy from the magnetic dipole and its effect on the parameter space allowed by the requirement of a strongly enough first order EWPhT. We then investigate the constraints from DM thermal relic density (section 6), DM direct detection (section 7), LHC data on Higgs signal strength (section 8), LEP data and electroweak precision test (section 9). The combined result is present in section 10. Finally, conclusions and some discussions are given in section 11. 


\section{Singlet fermionic dark matter model}

We consider an extension of the SM with a gauge singlet Dirac fermion $\psi$ which interacts with SM particles through a gauge singlet scalar $S$. The tree-level Higgs potential of this model is given by

$V(\Phi, S)=-\mu_{\phi}^{2} \Phi^{\dagger} \Phi+\lambda_{\phi}\left(\Phi^{\dagger} \Phi\right)^{2}-\mu_{1}^{3} S-\frac{1}{2} \mu_{s}^{2} S^{2}-\frac{1}{3} \mu_{3} S^{3}+\frac{1}{4} \lambda_{s} S^{4}+\mu \Phi^{\dagger} \Phi S+\frac{1}{2} \lambda \Phi^{\dagger} \Phi S^{2}$,

where $\Phi$ is the SM Higgs doublet

$$
\Phi=\left(\begin{array}{c}
G^{+} \\
\frac{1}{\sqrt{2}}\left(\phi^{0}-i G^{0}\right)
\end{array}\right),
$$

where $G^{ \pm}, G^{0}$ are the would-be Goldstone bosons. The coefficient $\mu_{1}$ in eq. (2.1) can be eleminated by a shift of the field $S, S \rightarrow S+\sigma$, which only causes a redefinition of parameters. In general both $\phi^{0}$ and $S$ can develop non-zero VEVs at zero temperature which are defined as $\left.\varphi_{0} \equiv\left\langle\phi^{0}\right\rangle\right|_{T=0}$ and $\left.s_{0} \equiv\langle S\rangle\right|_{T=0}$. The last two terms in eq. (2.1) lead to off-diagonal terms in the squared mass matrix of singlet scalar and the SM Higgs boson, which introduces a mixing between $\phi^{0}$ and $S$. The squared mass matrix of $\phi^{0}$ and $S$ is given by

$$
\mathcal{M}^{2}=\left(\begin{array}{ll}
\mathcal{M}_{11}^{2} & \mathcal{M}_{12}^{2} \\
\mathcal{M}_{21}^{2} & \mathcal{M}_{22}^{2}
\end{array}\right)
$$

where

$$
\begin{aligned}
& \mathcal{M}_{11}^{2}=-\mu_{\phi}^{2}+3 \lambda_{\phi} \varphi_{0}^{2}+\frac{1}{2} \lambda s_{0}^{2}+\mu s_{0}, \\
& \mathcal{M}_{22}^{2}=-\mu_{s}^{2}-2 \mu_{3} s_{0}+3 \lambda_{s} s_{0}^{2}+\frac{1}{2} \lambda \varphi_{0}^{2}, \\
& \mathcal{M}_{12}^{2}=\mathcal{M}_{21}^{2}=\mu \varphi_{0}+\lambda \varphi_{0} s_{0} .
\end{aligned}
$$

The squared mass matrix in eq. (2.3) can be diagonalized by rotating $\phi^{0}$ and $S$ into mass eigenstates $(h, H)$

$$
\left(\begin{array}{c}
h \\
H
\end{array}\right)=\left(\begin{array}{cc}
\cos \alpha & -\sin \alpha \\
\sin \alpha & \cos \alpha
\end{array}\right)\left(\begin{array}{c}
\phi^{0} \\
S
\end{array}\right),
$$

where the mixing angle $\alpha$ is

$$
\tan 2 \alpha=\frac{2 m_{12}^{2}}{\left(m_{22}^{2}-m_{11}^{2}\right)} .
$$

The value of $\alpha$ is defined in the range $0^{\circ}-45^{\circ}$, such that $h$ plays the role of the SM-like Higgs particle while $H$ is singlet dominant. The interaction involving the singlet fermionic DM particle $\psi$ is given by the Lagrangian

$$
\mathcal{L}_{\psi}=i \bar{\psi} \not \partial \psi-y_{\psi} \bar{\psi} \psi S \text {. }
$$

In general $S$ can develope a non-zero VEV, which contributes to the mass of the fermionic DM particle $\psi$. In this work we consider the case where $\psi$ only obtains mass from the VEV of $S$, namely $m_{\psi}=y_{\psi} s_{0}$, which makes the model more predictive. 


\section{Effective potential and EWPhT}

The tree-level potential for $\varphi=\left\langle\phi^{0}\right\rangle$ and $s=\langle S\rangle$ can be written as

$$
V_{0}(\varphi, s)=-\frac{1}{2} \mu_{\phi}^{2} \varphi^{2}-\frac{1}{2} \mu_{s}^{2} s^{2}-\frac{1}{3} \mu_{3} s^{3}+\frac{1}{2} \mu s \varphi^{2}+\frac{1}{4} \lambda_{\phi} \varphi^{4}+\frac{1}{4} \lambda_{s} s^{4}+\frac{1}{4} \lambda s^{2} \varphi^{2} .
$$

The coefficients $\mu_{\phi}$ and $\mu_{s}$ can be rewritten in terms of the VEVs $\varphi_{0}$ and $s_{0}$ according to the minimization conditions of the tree-level potential. However, the minimization conditions can not guarantee that $\left(\varphi_{0}, s_{0}\right)$ is the global minimum. Thus a check on whether there exists a deeper minimum is needed. In order to guarantee the stability of $\left(\varphi_{0}, s_{0}\right)$ as the global vacuum, it is also required that the potential is bounded-from-below.

The parameters $\lambda_{\phi}, \mu$ and $\lambda$ can be rewritten in terms of three physical parameters, i.e. the masses of the two Higgs particles $m_{h}, m_{H}$ and the mixing angle $\alpha$, as follows

$$
\begin{aligned}
\lambda_{\phi} & =\frac{1}{2 \varphi_{0}^{2}}\left(m_{h}^{2} \cos ^{2} \alpha+m_{H}^{2} \sin ^{2} \alpha\right), \\
\mu & =-2 \frac{s_{0}}{\varphi_{0}^{2}}\left(m_{h}^{2} \sin ^{2} \alpha+m_{H}^{2} \cos ^{2} \alpha+\mu_{3} s_{0}-2 \lambda_{s} s_{0}^{2}\right), \\
\lambda & =\frac{1}{\varphi_{0} s_{0}}\left[\left(m_{H}^{2}-m_{h}^{2}\right) \sin \alpha \cos \alpha-\mu \varphi_{0}\right] .
\end{aligned}
$$

We include one-loop Coleman-Weinberg correction of the potential at zero temperature [68]

$$
V_{1}(\varphi, s)=\frac{1}{64 \pi^{2}} \sum_{i} N_{i} m_{i}^{4}(\varphi, s)\left[\log \frac{m_{i}^{2}(\varphi, s)}{Q^{2}}-C_{i}\right],
$$

where $i$ runs over all the particles in the loop, and $N_{i}$ is the degrees of freedom of the particle $i, C_{i}$ is a constant ( $C_{i}=6 / 5$ for gauge bosons, $C_{i}=3 / 2$ for scalars and fermions), $Q$ is the renormalization scale which we fix at the mass of the top quark. The counter terms $V_{\mathrm{CT}}(\varphi, s)$ needed to renormalize the potential are given in appendix A.

The one-loop effective potential at finite temperature $T$ can be written as

$$
V_{\mathrm{eff}}(\varphi, s ; T)=V_{0}(\varphi, s)+V_{1}(\varphi, s)+V_{\mathrm{CT}}(\varphi, s)+V_{1}(\varphi, s ; T),
$$

where $V_{1}(\varphi, s ; T)$ is the one-loop thermal corrections

$$
V_{1}(\varphi, s ; T)=\frac{T^{4}}{2 \pi^{2}}\left[\sum_{i} n_{i} I_{\mathrm{B}}\left(a_{i}\right)+\sum_{j} n_{j} I_{\mathrm{F}}\left(a_{j}\right)\right],
$$

where $a=m^{2}(\varphi, s) / T^{2}, i(j)$ runs over all the bosons (fermions), $n_{i(j)}$ denotes the degrees of freedom of bosons (fermions), and $I_{\mathrm{B}(\mathrm{F})}(a)$ is defined as

$$
I_{\mathrm{B}(\mathrm{F})}(a)=\int_{0}^{\infty} d x x^{2} \ln \left(1 \mp e^{-\sqrt{x^{2}+a}}\right)
$$

where the sign $-(+)$ is for bosons (fermions). 

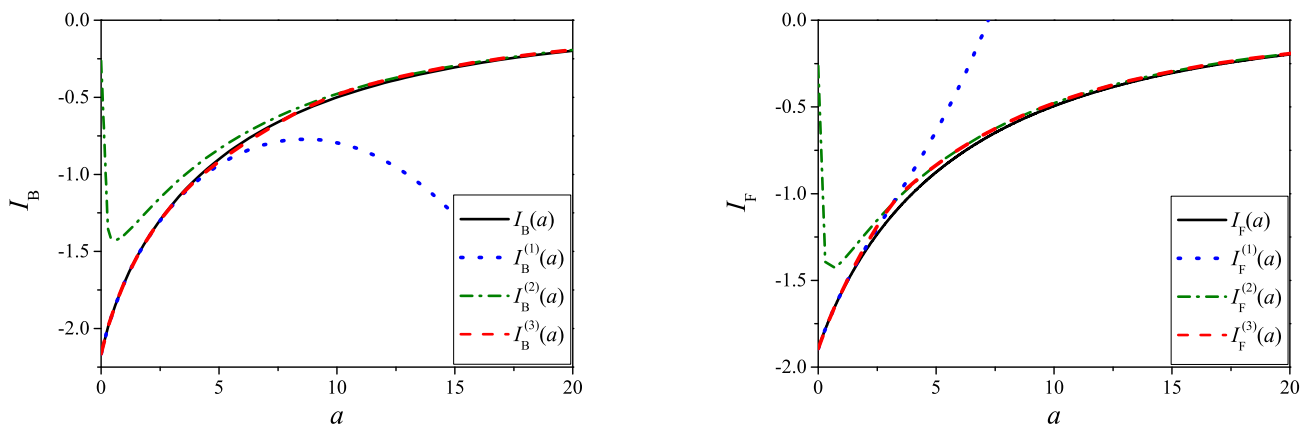

Figure 1. Left) Comparison of different approximations of the function $I_{\mathrm{B}}(a)$. The black solid and red dashed curves correspond to the numerical value and the approximation $I_{\mathrm{B}}^{(3)}(a)$, respectively. The blue dotted and green dot-dashed curves correspond to the high- and low-temperature approximations, respectively. Right) The same as left but for $I_{\mathrm{F}}(a)$.

Since the evaluation of the integration in eq. (3.6) is computationally expensive, it is necessary to have an analytical approximation. In the high temperature limit, i.e. $m(\varphi, s) / T \ll 1, I_{\mathrm{B}(\mathrm{F})}(a)$ can be expanded as [69]

$$
\begin{aligned}
& I_{\mathrm{B}}^{(1)}(a)=-\frac{\pi^{4}}{45}+\frac{\pi^{2}}{12} a-\frac{\pi}{6} a^{\frac{3}{2}}-\frac{1}{32} a^{2}\left[\log (a)-\gamma_{\mathrm{B}}\right], \\
& I_{\mathrm{F}}^{(1)}(a)=-\frac{7 \pi^{4}}{360}+\frac{\pi^{2}}{24} a+\frac{1}{32} a^{2}\left[\log (a)-\gamma_{\mathrm{F}}\right],
\end{aligned}
$$

where $\gamma_{\mathrm{B}}=5.40762$ and $\gamma_{\mathrm{F}}=2.63503$. The term cubic in $m / T$ in eq. (3.7) gives rise to the barrier in the potential which makes the phase transition first order. In the low temperature limit, $I_{\mathrm{B}(\mathrm{F})}(a)$ can be expanded as [11]

$$
I_{\mathrm{B}}^{(2)}(a ; n)=I_{\mathrm{F}}^{(2)}(a ; n)=-\sqrt{\frac{\pi}{2}} a^{\frac{3}{4}} e^{-a^{1 / 2}}\left(1+\frac{15}{8} a^{\frac{1}{2}}+\frac{105}{128} a\right) .
$$

The high- and low-temperature approximations are shown in figure 1. It can be seen that the high temperature approximation starts to fail when $a \gtrsim 3$. By matching the highand low-temperature approximations, we obtain a reasonable approximation to the integral

$$
I_{\mathrm{B}(\mathrm{F})}^{(3)}(a)=t_{\mathrm{B}(\mathrm{F})}(a) I_{\mathrm{B}(\mathrm{F})}^{(1)}(a)+\left(1-t_{\mathrm{B}(\mathrm{F})}(a)\right) I_{\mathrm{B}(\mathrm{F})}^{(2)}(a ; 2),
$$

where $t_{\mathrm{B}}(a)=e^{-(a / 6.3)^{4}}$ and $t_{\mathrm{F}}(a)=e^{-(a / 3.25)^{4}}$ are obtained by numerically fitting to the exact value of the integral. A comparison of different approximations of $I_{\mathrm{B}(\mathrm{F})}(a)$ is shown in figure 1. For the approximation $I_{\mathrm{B}(\mathrm{F})}^{(3)}(a)$ in eq. (3.10), the deviation to the exact value of $I_{\mathrm{B}(\mathrm{F})}$ is less than $5 \%$ in the region $0 \leqslant a \leqslant 20$.

The calculation of effective potential can be further improved by including thermal corrections to the boson masses which come from high order ring diagrams. After including the ring diagrams, the field-dependent squared mass matrix for the two Higgs particles is given by

$$
\mathcal{M}^{2}(\varphi, s ; T)=\left(\begin{array}{ll}
\mathcal{M}_{11}^{2} & \mathcal{M}_{12}^{2} \\
\mathcal{M}_{21}^{2} & \mathcal{M}_{22}^{2}
\end{array}\right)+\left(\begin{array}{cc}
c_{\phi} & 0 \\
0 & c_{s}
\end{array}\right) T^{2}
$$


where the matrix elements $\mathcal{M}_{i j}$ are defined analogously as in eq. (2.4) with replacements $\varphi_{0} \rightarrow \varphi, s_{0} \rightarrow s, c_{\phi}$ and $c_{s}$ are defined as

$$
\begin{aligned}
c_{\phi} & =\frac{1}{48}\left(9 g^{2}+3 g^{2}+12 y_{t}^{2}+24 \lambda_{\phi}+2 \lambda\right) \\
c_{s} & =\frac{1}{12}\left(2 \lambda+3 \lambda_{s}+2 y_{\psi}^{2}\right)
\end{aligned}
$$

where $y_{t}$ is the top Yukawa coupling, $g$ and $g^{\prime}$ are the $\mathrm{SU}(2)_{L}$ and $\mathrm{U}(1)_{Y}$ gauge couplings, respectively. The thermal masses of the Goldstone bosons are given by

$$
m_{G^{0}, G^{ \pm}}^{2}(\varphi, s ; T)=-\mu_{\phi}^{2}+\lambda_{\phi} \varphi^{2}+\mu s+\frac{1}{2} \lambda s^{2}+c_{\phi} T^{2} .
$$

In order to trigger first order EWPhT, the thermal effective potential must have two degenerate minima separated by a barrier at the critical temperature. Due to the existence of the extra scalar field, there can exist two kinds of barriers in this model

- Tree-level barrier. This kind of barrier arises from the terms linear and cubic in $s$ which are already present in the effective potential at tree-level. In the scenario with tree-level barrier only, one important implication is that a first order EWPhT is always related to a change of the VEV of the singlet scalar field at the critical temperature. If the VEV of the singlet scalar field is constant during the EWPhT, the tree-level potential would have the same structure as that in the SM case which has no barrier.

- Loop-level barrier. This kind of barrier arises from the term cubic in $m / T$ which comes from the thermal one-loop corrections of the bosonic fields to the effective potential. It also exists in the SM case, which is however not enough to trigger a strongly first order EWPhT. In this model, the extra singlet scalar field can contribute to this kind of barrier and make it possible to trigger a strongly first order EWPhT.

For the investigation of the tree-level barrier, it is enough to keep only the leading order terms which are quadratic in $m / T$ of the high-temperature approximation

$$
V_{1}^{\mathrm{lo}}(\varphi, s ; T)=\left(\frac{1}{2} \kappa_{\phi} \varphi^{2}+\frac{1}{2} \kappa_{s} s^{2}+\kappa_{3} s\right) T^{2}
$$

where

$$
\begin{aligned}
\kappa_{\phi} & =\frac{1}{48}\left(9 g^{2}+3 g^{\prime 2}+12 y_{t}^{2}+24 \lambda_{\phi}+2 \lambda\right) \\
\kappa_{s} & =\frac{1}{12}\left(2 \lambda+3 \lambda_{s}+2 y_{\psi}^{2}\right) \\
\kappa_{3} & =\frac{1}{12}\left(-\mu_{3}+2 \mu\right) .
\end{aligned}
$$

For an illustration of the tree-level barrier, we use $V_{0}(\varphi, s)+V_{1}^{\text {lo }}(\varphi, s ; T)$ as an approximation of the effective potential. The stationary points of this effective potential 
are located at the intersections of the curves determined by $\partial V_{\text {eff }}(\varphi, s ; T) / \partial \varphi=0$ and $\partial V_{\text {eff }}(\varphi, s ; T) / \partial s=0$ which lead to

$$
\varphi=0 \quad \text { or } \quad \varphi^{2}=f_{h}(s)=-\frac{\kappa_{\phi} T^{2}-\mu_{\phi}^{2}+\mu s+\frac{1}{2} \lambda s^{2}}{\lambda_{\phi}}
$$

and

$$
\varphi^{2}=f_{s}(s)=-2 \cdot \frac{\kappa_{3} T^{2}+\left(\kappa_{s} T^{2}-\mu_{s}^{2}\right) s-\mu_{3} s^{2}+\lambda_{s} s^{3}}{\mu+\lambda s} .
$$

We show the evolution of this effective potential with temperature in figure 2. Since at sufficiently high temperature the effective potential is dominated by the contributions from the thermal corrections in eq. (3.15), there is only one minimum at $\varphi=0$, as shown in figure 2(a). As the temperature decreases, local minimum with $\varphi \neq 0$ appears, but the original minimum at $\varphi=0$ is still the global one. At the critical temperature $T_{c}$, the minimum at $\varphi=\varphi_{c}$ becomes degenerate with the minimum at $\varphi=0$, as shown in figure 2(c). The minimum at $\varphi=0$ becomes meta-stable and the phase transition of $\varphi$ occurs. It can be seen that there is a barrier which separates the two degenerate minima and leads to first order EWPhT. After the phase transition of $\varphi$, the local minimum at $\varphi \neq 0$ becomes the global one, as shown in figure $2(\mathrm{e})$.

\section{Parameter space for EWPhT}

To check whether a EWPhT is strongly first order, we should first find the critical temperature which is defined as when there appear two degenerate minima. We search for $T_{c}$ in the range from $T_{\min }=1 \mathrm{GeV}$ to $T_{\max }=1 \mathrm{TeV}$. We start from $T_{\min }$, then increase the temperature and check the minima of the potential. The critical temperature is obtained when the local minimum at $\varphi \neq 0$ becomes degenerate with the one at $\varphi=0$. If the global minimum at $T_{\max }$ is at $\varphi \neq 0$, EWPhT will not occur.

When the EWPhT occurs, there is a path connecting the two degenerate local minima which has the lowest barrier (see figure 2(c)). If there is no barrier along this path, the EWPhT is of the second order. In this case the local minimum corresponds to a flat direction of the potential. To identify this case we follow the method in ref. [64] to check whether a putative minimum is a real minimum. We minimize the potential on small circles surrounding the putative local minimum. If the minima on the circles are greater than the putative minimum, it is indeed a true local minimum.

We explore the full parameter space of the singlet fermionic DM model which includes: $m_{H}, \alpha, s_{0}, \mu_{3}, \lambda_{s}$, and $m_{\psi}$. We scan these parameters in the ranges

$$
\begin{aligned}
& 10 \mathrm{GeV} \leqslant m_{H} \leqslant 1 \mathrm{TeV}, \quad 0 \leqslant \alpha \leqslant 45^{\circ}, \quad-1 \mathrm{TeV}<s_{0} \leqslant 1 \mathrm{TeV}, \\
& -1 \mathrm{TeV} \leqslant \mu_{3} \leqslant 1 \mathrm{TeV}, \quad 0 \leqslant \lambda_{s} \leqslant 3, \quad-3 \leqslant y_{\psi} \leqslant 3 .
\end{aligned}
$$

The mass of the SM-like Higgs particle is fixed at $m_{h}=125 \mathrm{GeV}$.

We use an improved random walk sampling algorithm to scan the parameter space based on a MCMC method with the Metropolis algorithm. The likelihood of a given 


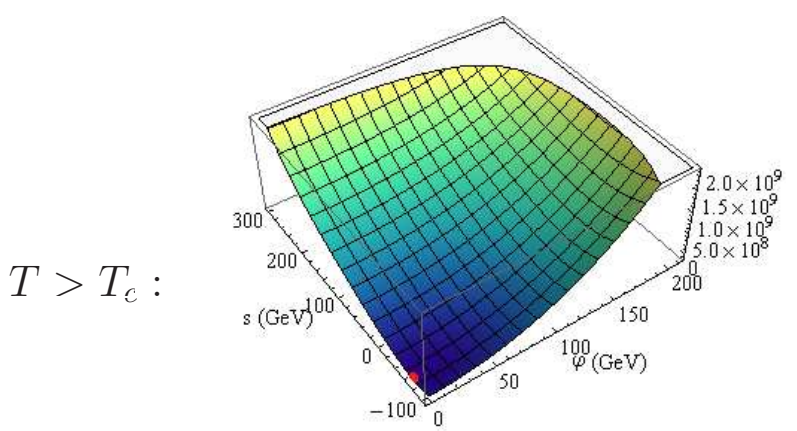

(a)

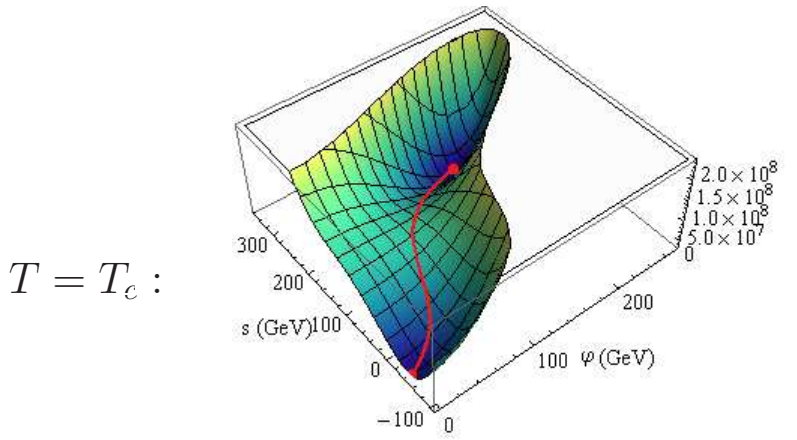

(c)

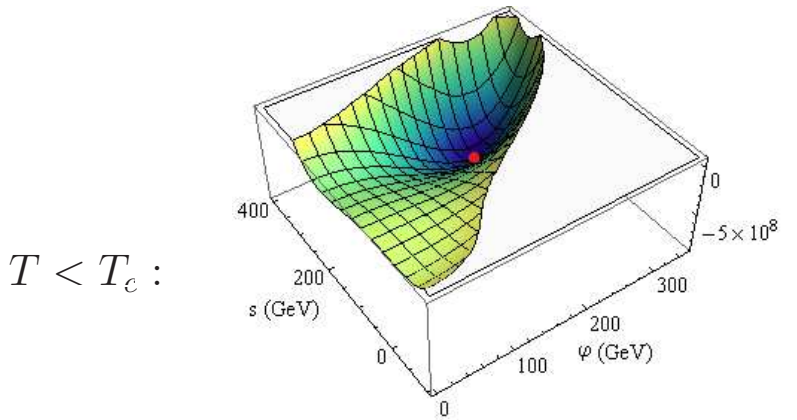

(e)

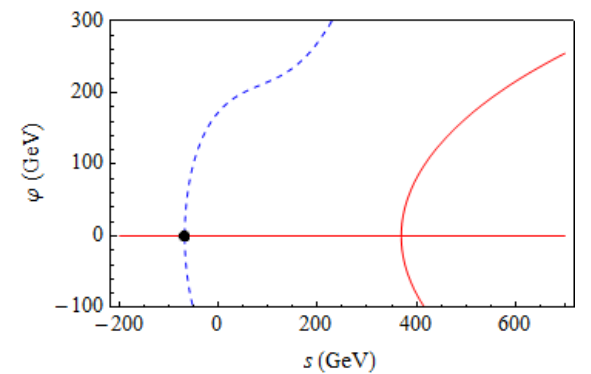

(b)

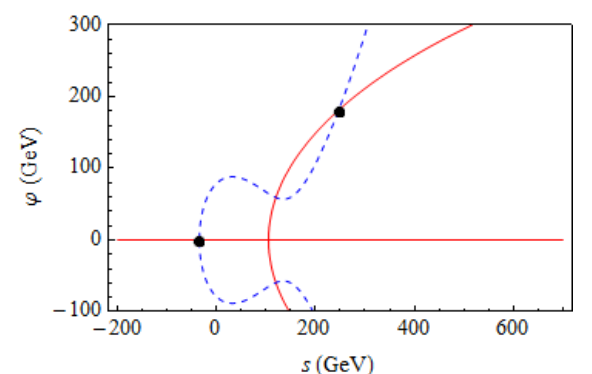

(d)

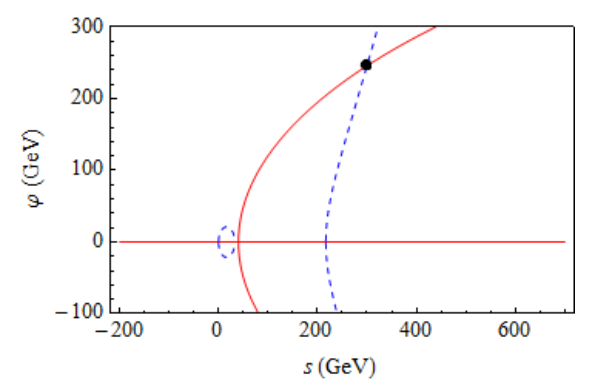

(f)

Figure 2. Thermal evolution of the effective potential in the senario with tree-level barrier only. The parameters are fixed at $s_{0}=300 \mathrm{GeV}, \lambda_{\phi}=1, \mu_{3}=250 \mathrm{GeV}, \lambda_{s}=1, \mu=-250 \mathrm{GeV}, \lambda=0.1$ and $y_{\psi}=0.5$. Left) The effective potentials at $T>T_{c}, T=T_{c}$, and $T<T_{c}$ from top to bottom, respectively. The global minima of the effective potentials are indicated by red dots. In (c) the path with lowest barrier between the two local minima is indicated by the red line. Right) Curves corresponding to $\partial V_{\text {eff }}(\varphi, s ; T) / \partial \varphi=0$ (solid line) and $\partial V_{\text {eff }}(\varphi, s ; T) / \partial s=0$ (dashed line). The global minima are located at the intersections of the two curves as indicated by the black dots.

parameter set $\boldsymbol{x}$ is defined as

$$
\mathscr{L}(\boldsymbol{x})=\min \left\{\varphi_{c} / T_{c}, 1\right\} .
$$

We run multi-chain samplers with initial values uniformly distributed in the 6-dimensional parameter space and obtain a sample set containing about $5 \times 10^{6}$ sample points satisfying $\varphi_{c} / T_{c}>1$. 


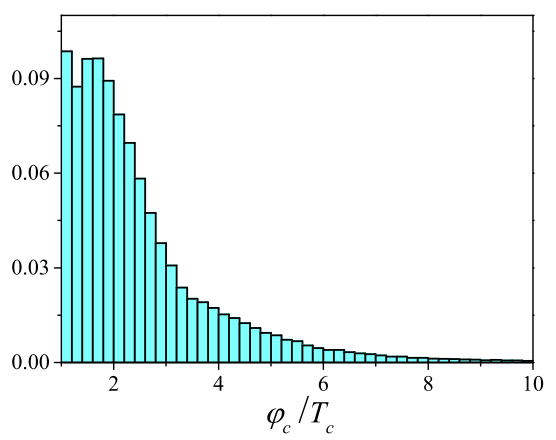

Figure 3. The relative frequency distribution of the order parameter $\varphi_{c} / T_{c}$ of the samples satisfying $\varphi_{c} / T_{c}>1$ which are obtained using the likelihood function in eq. (4.2).
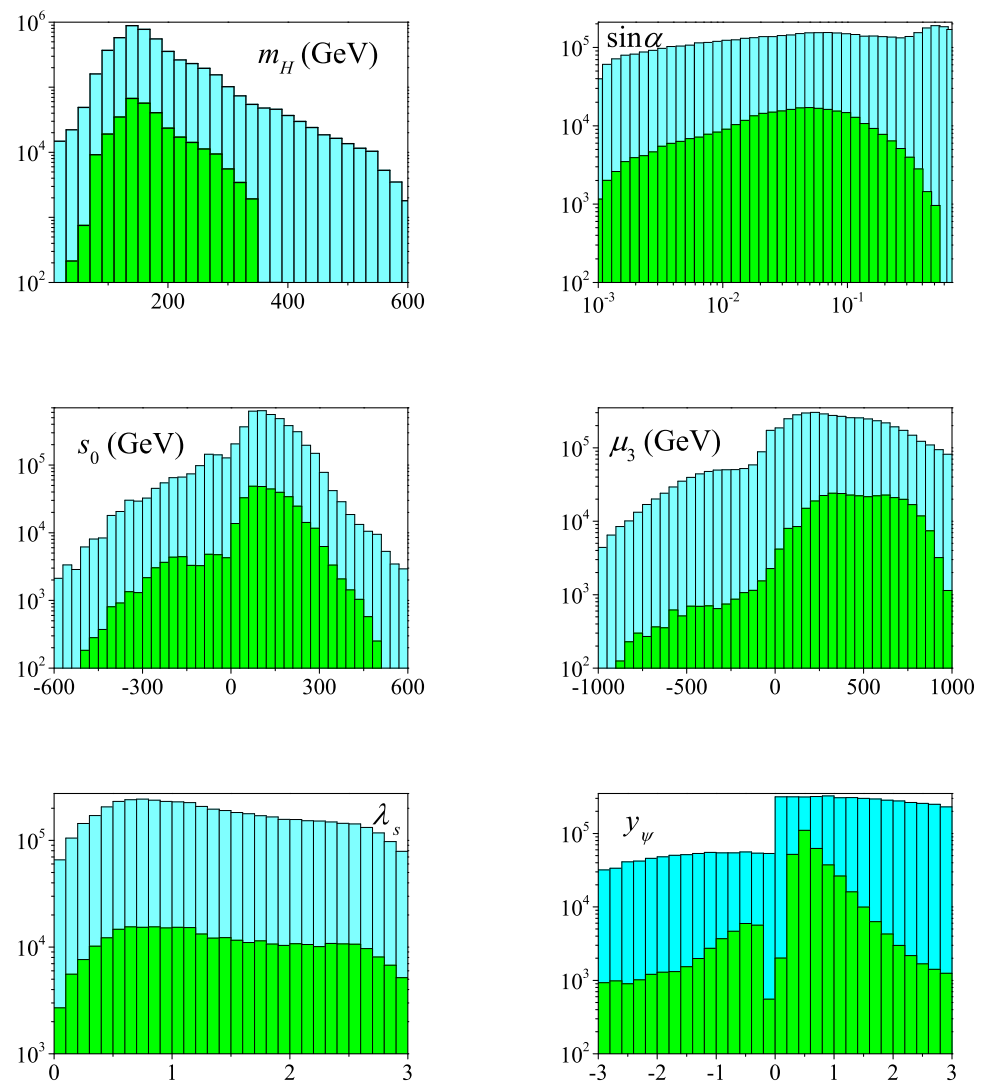

Figure 4. The frequency distributions of the free parameters $m_{H}, \sin \alpha, s_{0}, \mu_{3}, \lambda_{s}$ and $y_{\psi}$ of the samples satisfying $\varphi_{c} / T_{c}>1$ (cyan areas) which are obtained using the likelihood function in eq. (4.2). The green areas are the distributions after considering all the constraints from the observables such as DM thermal relic density, DM-nucleon cross section, Higgs signal strength, Higg- $Z-Z$ coupling strength and the oblique parameters. 


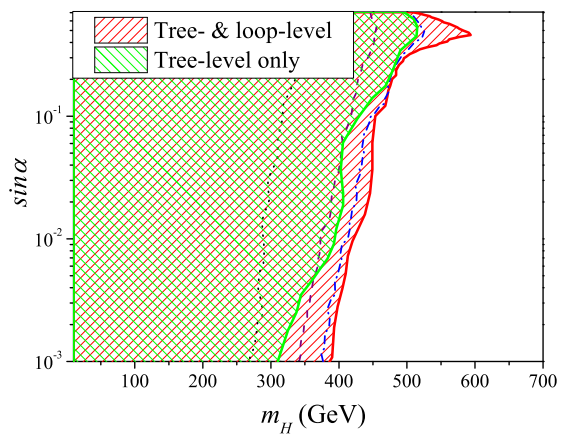

(a)

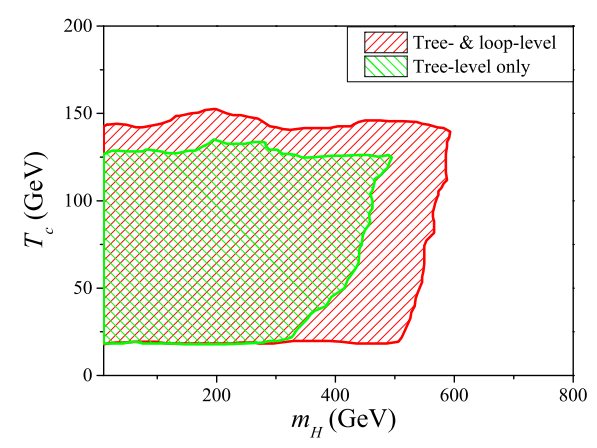

(b)

Figure 5. Comparison between the allowed regions in the $m_{H}-\sin \alpha$ (left) and $m_{H}-T_{c}$ (right) planes for the case with tree-level barrier only and the case with both tree- and loop-level barriers. In the left panel, the lines indicate the boundary of the allowed parameter space for $\varphi_{c} / T_{c}>\mathcal{E}$ including both tree- and loop-level barriers with $\mathcal{E}=1.2$ (blue dot-dashed), $\mathcal{E}=1.5$ (purple dashed) and $\mathcal{E}=2$ (black dotted), respectively.

The relative frequency distribution of the order parameter $\varphi_{c} / T_{c}$ is shown in figure 3 . Strongly first order EWPhTs are found with $\varphi_{c} / T_{c}$ up to 10 in this model. The frequency distributions of the 6 free parameters are shown in figure 4 . It can be seen that, for $\mathcal{E}=1$, there exists an upper limit on the mass of the second Higgs particle around $600 \mathrm{GeV}$, and $s_{0}$ is constrained to $\left|s_{0}\right| \lesssim 600 \mathrm{GeV}$. Heavier particles cannot trigger a strongly enough first order EWPhT, as the contributions of heavy particles suffer from exponential suppression as shown in eq. (3.9).

In this model, the extra scalar field leads to a tree-level barrier at the critical temperature. Both of the tree- and the loop-level barriers can trigger strongly first order EWPhT. A comparison between the tree- and loop-level barriers is shown in figure 5 in which we plot the allowed regions for the case with tree-level barrier only and the case with both tree- and loop-level barriers. As shown by the figure, the allowed region with both the treeand loop-level barriers is larger than that in tree-level only case. For instance, the upper limits of $m_{H}$ is about $100 \mathrm{GeV}$ higher at $\sin \alpha=0.001$ for $\mathcal{E}=1$. The loop-level cubic terms also raise the critical temperature. As shown in figure $5(\mathrm{~b})$, the critical temperature has an upper limit around $150 \mathrm{GeV}$, which is about $10 \%$ lower in the case where only the tree-level barrier is considered.

\section{The effect of the sphaleron magnetic moment}

The condition for the sphaleronic interactions to be sufficiently suppressed to preserve the baryon asymmetry generated during the EWPhT is given by $[8,9]$

$$
\frac{E_{s p h}\left(T_{c}, B\right)}{T_{c}} \gtrsim 35
$$


In the SM, the sphaleron energy relates to $\varphi$ the VEV of the Higgs field by

$$
E_{\mathrm{sph}}=\frac{8 \pi m_{W}(\varphi)}{g^{2}} \mathcal{C}
$$

where $m_{W}(\varphi)$ is the $W$-Boson mass, and $\mathcal{C} \sim 2$ is a constant determined by the sphaleron solution. Thus, this condition can be translated into eq. (1.1) with $\mathcal{E}=1$. This conclusion can be modified if there exists a primordial magnetic field in the early universe [7, 70]. The magnetic field can be generated before or during the EWPhT through various mechanisms (for a review see ref. [71]). Meanwhile, the electroweak sphaleron may develope a $\mathrm{U}(1)_{Y}$ magnetic dipole moment. The interaction between the magnetic dipole and the background magnetic field can give negative contribution to the sphaleron energy. Consequently, the preservation of the baryon asymmetry requires a larger value of $\mathcal{E}$.

In the presence of a background hypermagnetic field $B$, the sphaleron magnetic moment $\mu$ can lower the sphaleron energy

$$
E_{\mathrm{sph}}(T, B)=E_{\mathrm{sph}}(T)-\mu(T) B .
$$

In this work, we parametrize the external hypermagnetic field as [7]

$$
B=b T^{2},
$$

where $b$ is a dimensionless parameter which is usually taken to be $b \lesssim 0.4$. To estimate the effect of sphaleron dipole moment in this model, we follow the approach adopted in refs. $[7,70]$. The formulas which give the sphaleron solution and the sphaleron magnetic moment are summarized in the appendix $\mathrm{C}$.

In this model, the relation between $E_{\mathrm{sph}} /\left(35 T_{c}\right)$ and $\varphi_{c} / T_{c}$ is complicated and can only be calculated numerically. In table 1 we show the values of $E_{\mathrm{sph}} /\left(35 T_{c}\right)$ and $\varphi_{c} / T_{c}$ for several typical parameter sets. It can be seen that the presence of the sphaleron magnetic moment can lower the sphaleron energy, which makes the value of $E_{\mathrm{sph}} /\left(35 T_{c}\right)$ lower than the value of $\varphi_{c} / T_{c}$. However, the difference between them are within $10 \%$. As can be seen in table 1 , in the listed parameter sets, the values of $\varphi_{c} / T_{c}$ varies from 1.2 to 2.12 , and all of the parameter sets can provide a strongly enough first order EWPhT. This is different from the conclusion in the case of the SM where the inclusion of the magnetic moment generally requires $\varphi_{c} / T_{c} \gtrsim 1.3$ [70]. The reason is that the extra scalar field $S$ in this model raises the sphaleron energy but gives no contribution to the sphaleron magnetic moment, which weakens the contribution from the sphaleron magnetic moment. In figure 5(a) and figure 8 , we show the boundary of the allowed parameter space for $\mathcal{E}=1.2$. It can be seen in figure 8 that, after considering all the constrains from observables, the difference between the upper bound on the mass of the second Higgs particle for $\mathcal{E}=1.2$ and that for $\mathcal{E}=1$ is within $10 \mathrm{GeV}$.

\section{$6 \quad$ DM thermal relic density}

The fermionic DM particle $\psi$ can annihilate into final states $\bar{f} f, W^{+} W^{-}, Z Z, h h, H H$ or $h H$ via $s$-channel Higgs particle exchanges. For annihilation with final states $h h, H H$ or 


\begin{tabular}{|ccccccccc|}
\hline$m_{2}$ & $\sin \alpha$ & $s_{0}$ & $\mu_{3}$ & $\lambda_{s}$ & $E_{\mathrm{sph}}\left(T_{c}\right)$ & $E_{\text {dipole }}\left(T_{c}, B\right)$ & $\frac{\varphi_{c}}{T_{c}}$ & $\frac{E_{\mathrm{sph}}\left(T_{c}, B\right)}{35 T_{c}}$ \\
\hline 256.8 & 0.05 & 42.7 & 125.8 & 1.05 & 1.16 & 0.08 & 1.20 & 1.14 \\
120.0 & 0.074 & 136.6 & 267.6 & 0.75 & 1.39 & 0.05 & 2.12 & 2.08 \\
97.2 & 0.002 & 212.8 & 235.5 & 0.70 & 0.90 & 0.06 & 1.21 & 1.16 \\
197.1 & 0.14 & 100.2 & 464.1 & 0.92 & 1.33 & 0.04 & 1.65 & 1.62 \\
127.2 & 0.02 & 118.8 & 61.8 & 0.80 & 1.18 & 0.05 & 1.38 & 1.34 \\
\hline
\end{tabular}

Table 1. Sphaleron and magnetic dipole energies for several typical parameter sets. The sphaleron energy and magnetic dipole energy are in units of $4 \pi \sqrt{\varphi_{c}^{2}+s_{c}^{2}} / g$. Other parameters $m_{2}, s_{0}$ and $u_{3}$ are in unit of $\mathrm{GeV}$. The SM Higgs mass is set to $m_{1}=125 \mathrm{GeV}$. The magnetic field is fixed at $B=0.4 T^{2}$.
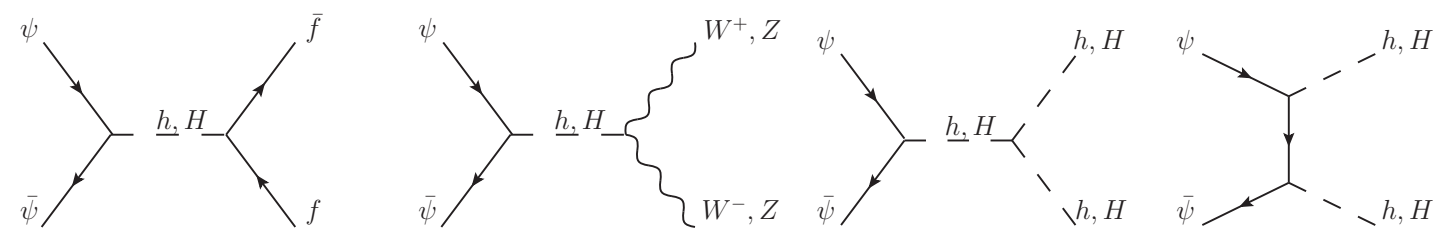

Figure 6. Feynman diagrams for the annihilation of fermionic DM particle.

$h H$, the $t$ - and $u$-channels are also possible. The Feynman diagrams for these processes are shown in figure 6 . The cross sections for these processes are given in appendix B.

The thermal average of the cross section multiplied by the DM relative velocity $v_{\text {rel }}$ at a temperature $T$ is given by

$$
\left\langle\sigma v_{\mathrm{rel}}\right\rangle=\frac{1}{8 m_{\psi}^{4} T K_{2}^{2}\left(m_{\psi} / T\right)} \int_{4 m_{\psi}^{2}}^{\infty} d \mathfrak{s} \sigma(\mathfrak{s})\left(\mathfrak{s}-4 m_{\psi}^{2}\right) \sqrt{\mathfrak{s}} K_{1}\left(\frac{\sqrt{\mathfrak{s}}}{T}\right)
$$

where $K_{1}\left(K_{2}\right)$ is the modified Bessel function of the first (second) kind, $\sqrt{\mathfrak{s}}$ denotes the center-of-mass energy. The temperature evolution of the abundance $Y$ which is defined as the number density devided by the entropy density of the DM particle is governed by the Boltzmann equation [72]

$$
\frac{d Y}{d T}=\sqrt{\frac{\pi g_{*}(T)}{45}} M_{\mathrm{pl}}\left\langle\sigma v_{\mathrm{rel}}\right\rangle\left[Y(T)^{2}-Y_{\mathrm{eq}}(T)^{2}\right]
$$

where $M_{\mathrm{pl}}=1.22 \times 10^{19} \mathrm{GeV}$ is the Planck mass scale, $g_{*} 1$ is the effective number of relativistic degrees of freedom, and $Y_{\mathrm{eq}}$ is the abundance at equilibrium. The relic density is related to the present-day abundance $Y\left(T_{0}\right)$ by

$$
\Omega h^{2}=2.472 \times 10^{8} \mathrm{GeV}^{-1} m_{\psi} Y\left(T_{0}\right),
$$

where $T_{0}$ is the temperature of the microwave background. In this work we adopt the freeze-out approximation, and use micrOMEGAs3.3 for numerical calculation of the relic density $[73,74]$. The freeze-out temperature $T_{f}$ can be defined from the relation $Y\left(T_{f}\right)=$ 


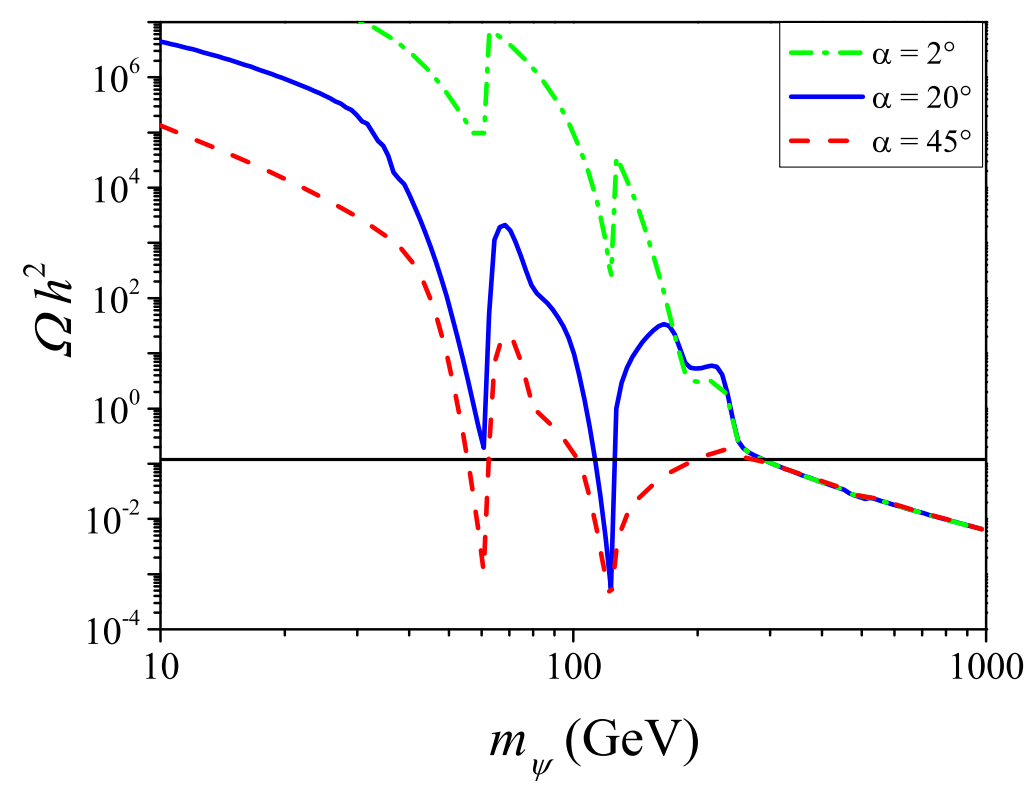

Figure 7. DM thermal relic density as a function of the DM particle mass with $m_{H}=250 \mathrm{GeV}$ for different values of $\alpha=2^{\circ}, 20^{\circ}$ and $45^{\circ}$, respectively. Other parameters are fixed at $s_{0}=300 \mathrm{GeV}$, $\mu_{3}=300 \mathrm{GeV}$ and $\lambda_{s}=1$. The horizontal solid line indicates $\Omega h^{2}=0.1187$ [17].

$(1+\delta) Y_{\text {eq }}\left(T_{f}\right)$ with $\delta$ being a constant and can be determined by solving

$$
\left.\frac{d \ln Y_{\mathrm{eq}}}{d T}\right|_{T=T_{f}}=\delta(\delta+2) \sqrt{\frac{\pi g_{*}\left(T_{f}\right)}{45}} M_{\mathrm{pl}}\left\langle\sigma v_{\mathrm{rel}}\right\rangle Y_{\mathrm{eq}}\left(T_{f}\right)
$$

with $\delta=1.5$ [73]. Below the freeze-out temperature, $Y_{\text {eq }} \ll Y$, eq. (6.2) can be integrated

$$
\frac{1}{Y\left(T_{0}\right)}=\frac{1}{Y\left(T_{f}\right)}+\sqrt{\frac{\pi}{45}} M_{\mathrm{pl}} \int_{T_{0}}^{T_{f}} \sqrt{g_{*}(T)}\left\langle\sigma v_{\mathrm{rel}}\right\rangle d T .
$$

The deviation of this approximation from the exact solution of the Boltzmann equation eq. (6.2) is within $2 \%$ [73].

Figure 7 shows the thermal relic density as a function of the DM particle mass. Since the measurement on the DM relic density from the Planck experiment is very precise, the value of $m_{\psi}$ can actually be solved from the DM relic density up to a five-fold ambiguity. The ambiguity arises from the two resonant annihilations when $m_{\psi} \approx m_{h, H} / 2$.

\section{Direct detection of DM}

For a Dirac DM particle the spin-independent DM-proton elastic scattering cross section is given by

$$
\sigma_{\mathrm{SI}} \approx \frac{m_{r}^{2}}{\pi} \lambda_{p}^{2}
$$


where $m_{r}$ is the DM-proton reduced mass $m_{r}=m_{\psi} m_{p} /\left(m_{\psi}+m_{p}\right)$ with $m_{p}$ the proton mass. The coupling $\lambda_{p}$ is given by

$$
\frac{\lambda_{p}}{m_{p}}=\sum_{q=u, d, s} f_{T_{q}}^{(p)} \frac{\lambda_{q}}{m_{q}}+\frac{2}{27} f_{T_{g}}^{(p)} \sum_{q=c, b, t} \frac{\lambda_{q}}{m_{q}} .
$$

The coupling $\lambda_{q}$ at quark level in this model is

$$
\frac{\lambda_{q}}{m_{q}}=\frac{y_{\psi} \sin \alpha \cos \alpha}{\varphi_{0}}\left(\frac{1}{m_{h}^{2}}-\frac{1}{m_{H}^{2}}\right) .
$$

The parameters $f_{T_{q}}^{(p)}$ are defined from the nucleon matrix elements $m_{p} f_{T_{q}}^{(p)} \equiv\left\langle p\left|m_{q} \bar{q} q\right| p\right\rangle$ for $q=u, d, s$ and $f_{T_{g}}^{(p)}=1-\sum_{q=u, d, s} f_{T_{q}}^{(p)}$. In numerical calculations we take the values $f_{T_{u}}^{(p)}=0.0153, f_{T_{d}}^{(p)}=0.0191$ and $f_{T_{s}}^{(p)}=0.0447$ [75]. For some of the recent studies of these parameters we refer to the refs. [76-78].

Currently the strongest upper limits on $\sigma_{\mathrm{SI}}$ are given by the LUX experiment [66]. The allowed region in the $m_{H}-\sin \alpha$ plane is shown in figure 8 . It can be seen that the mixing angle is severely constrained by the LUX data, for instance $\sin \alpha \lesssim 0.1$ leading to $\alpha \lesssim 5.7^{\circ}$ at $m_{H}=350 \mathrm{GeV}$. In the region where $\left|m_{H}-m_{h}\right| \lesssim 20 \mathrm{GeV}$, the constraint from LUX data is significantly relaxed due to the destructive interference between the contributions from the two Higgs particles, as shown in eq. (7.3). In figure 8 we also show the upper bound on the mixing angle corresponding to the data of the XENON100 experiment. It can be seen that the XENON100 constraint on the mixing angle is much weaker than the LUX constraint, for instance $\sin \alpha \lesssim 0.4$ leading to $\alpha \lesssim 23^{\circ}$ at $m_{H}=350 \mathrm{GeV}$.

The next generation of DM direct detection experiments can push the upper bound on $\sigma_{\mathrm{SI}}$ down to $\sim 10^{-47} \mathrm{~cm}^{2}$ [79]. This upper bound can further constrain the mixing angle $\alpha$. In figure 8 , we show the upper bound on the mixing angle which corresponds to the projected exclusion limit of the future XENON1T experiment. It can be seen that $\sin \alpha$ can be further constrained to one order of magnitude lower than the upper bound from the LUX data in the regions off resonance, for instance $\sin \alpha \lesssim 0.01$ leading to $\alpha \lesssim 0.57^{\circ}$ at $m_{H}=350 \mathrm{GeV}$.

\section{Higgs signal strength at the LHC}

The LHC experiment has reported the discovery of a SM-like Higgs boson [15, 16]. Throughout our work we take the SM-like Higgs particle mass fixed at $m_{h}=125 \mathrm{GeV}$. The Higgs signal strengths in different channels such as $\bar{b} b, \tau^{+} \tau^{-}, \gamma \gamma, W W^{*}$ and $Z Z^{*}$ have been measured by the ATLAS, CMS and CDF experiments. The combined result on the Higgs signal strength with respect to the SM value shows no significant deviation from the SM prediction [80]

$$
r_{h}=1.02_{-0.12}^{+0.11}
$$

with $r_{h}$ defined as the signal strength of the SM-like Higgs particle in new physics models relative to that in the SM. We consider $r_{h}$ in the range $0.78-1.24$ which corresponds to the approximately $95 \%$ confidence level (CL) allowed range. 
The signal strength of the SM-like Higgs particle in this model with respect to the SM value is given by

$$
r_{h}=\frac{\sigma_{g g \rightarrow h} B_{h \rightarrow X X}}{\sigma_{g g \rightarrow h}^{\mathrm{SM}} B_{h \rightarrow X X}^{\mathrm{SM}}}=\frac{\sigma_{g g \rightarrow h}}{\sigma_{g g \rightarrow h}^{\mathrm{SM}}} \times \frac{\Gamma_{h \rightarrow X X}}{\Gamma_{h \rightarrow X X}^{\mathrm{SM}}} \times \frac{\Gamma_{h}^{\mathrm{SM}}}{\Gamma_{h}},
$$

where $X$ stands for any final state particle, $\sigma_{g g \rightarrow h}$ is the production cross section through gluon-gluon fusion of the SM-like Higgs particle, $\Gamma_{h \rightarrow X X}$ is the width of the SM-like Higgs particle decaying to $X, \Gamma_{h}$ is the total decay width of the SM-like Higgs particle, and $\sigma_{g g \rightarrow h}^{\mathrm{SM}}, \Gamma_{h \rightarrow X X}^{\mathrm{SM}}$ and $\Gamma_{h}^{\mathrm{SM}}$ are the corresponding values in the SM.

The mixing between the two Higgs particles leads to a universal $\cos \alpha$ suppression of all the couplings between the SM-like Higgs particle and the SM fermions and gauge bosons, which leads to

$$
\frac{\sigma_{g g \rightarrow h}}{\sigma_{g g \rightarrow h}^{\mathrm{SM}}}=\frac{\Gamma_{h \rightarrow X X}}{\Gamma_{h \rightarrow X X}^{\mathrm{SM}}}=\cos ^{2} \alpha .
$$

Additionally, the signal strength of the SM-like Higgs particle is also suppressed by two possible new invisible decay channels which are $h \rightarrow \bar{\psi} \psi$ and $h \rightarrow H H$. The total decay width of the SM-like Higgs particle in this model can be written as

$$
\Gamma_{h}=\Gamma_{h}^{\mathrm{SM}} \cos ^{2} \alpha+\Gamma_{h \rightarrow \bar{\psi} \psi}+\Gamma_{h \rightarrow H H}
$$

where $\Gamma_{h \rightarrow \bar{\psi} \psi}$ and $\Gamma_{h \rightarrow H H}$ are the decay widths of the SM-like Higgs particle via the two new channels

$$
\begin{aligned}
\Gamma_{h \rightarrow \bar{\psi} \psi} & =\frac{y_{\psi}^{2} m_{h}}{8 \pi} \beta_{\psi}^{3} \cdot \sin ^{2} \alpha, \\
\Gamma_{h \rightarrow H H} & =\frac{\lambda_{h H H}^{2}}{8 \pi m_{h}} \beta_{H},
\end{aligned}
$$

where $\beta_{\psi(H)}=\sqrt{1-4 m_{\psi(H)}^{2} / m_{h}^{2}}$ and $\lambda_{h H H}$ is the coupling of $h H H$ defined in eq. (B.14) in appendix B. Thus, the signal strength of the SM-like Higgs particle can be written as

$$
r_{h}=\frac{\Gamma_{h}^{\mathrm{SM}} \cos ^{4} \alpha}{\Gamma_{h}^{\mathrm{SM}} \cos ^{2} \alpha+\Gamma_{h \rightarrow \bar{\psi} \psi}+\Gamma_{h \rightarrow H H}} .
$$

Note that the signal strength $r_{h}$ is suppressed by $\cos ^{2} \alpha$ even if the two new invisible decay channels are kinematically forbidden.

In the parameter region where $m_{H}<m_{h} / 2, \Gamma_{h \rightarrow H H}$ is still considerably large even if the mixing angle is very small. In the limit without mixing between the two Higgs particles, it is given by

$$
\Gamma_{h \rightarrow H H}=\frac{\lambda^{2} \varphi_{0}^{2}}{16 \pi m_{h}} \beta_{H},
$$

which results in a constraint on the parameter $\lambda$

$$
\lambda^{2} \lesssim 14.2 \frac{m_{h} \Gamma_{h}^{\mathrm{SM}}}{\varphi_{0}^{2} \beta_{H}} .
$$


In the parameter region where $m_{H} \lesssim 30 \mathrm{GeV}$, this constraint is strong enough to exclude all the sample points, as shown in figure 8 .

Analogously, the signal strength of the second Higgs particle is given by

$$
r_{H}=\frac{\Gamma_{H}^{\mathrm{SM}} \sin ^{4} \alpha}{\Gamma_{H}^{\mathrm{SM}} \sin ^{2} \alpha+\Gamma_{H \rightarrow \bar{\psi} \psi}+\Gamma_{H \rightarrow h h}} .
$$

The signal strength of the second Higgs particle is proportional to $\sin ^{2} \alpha$, which comes from the coupling between the second Higgs particle and the SM fermions and gauge bosons, and it is also suppressed by the decay channels $H \rightarrow \bar{\psi} \psi$ and $H \rightarrow h h$.

The allowed region in the $m_{H}-\sin \alpha$ plane under this constraint is plotted in figure 8. It can be seen that the result on the signal strength of the SM-like Higgs particle imposes an upper bound on the mixing angle, due to the suppression factor $\cos ^{2} \alpha$ in the signal strength in eq. (8.7). When the invisible decay of the SM-like Higgs particle through the channel $h \rightarrow H H$ is kinematically forbidden, i.e. $m_{H}>m_{h} / 2$, the upper limit on the mixing angle is directly given by $\sin ^{2} \alpha \lesssim 0.22$, leading to $\alpha \lesssim 28^{\circ}$. When the channel $h \rightarrow H H$ is opened, i.e. $m_{H}<m_{h} / 2$, the mixing angle is further constrained, for instance $\sin \alpha \lesssim 0.01$ leading to $\alpha \lesssim 0.57^{\circ}$ at $m_{H}=50 \mathrm{GeV}$.

Besides the constraint on the signal strength of the SM-like Higgs particle, the current LHC data also set an upper bound on a Higgs particle with a mass larger than $145 \mathrm{GeV}$ [81], which can be translated into an upper bound on $r_{H}$ in this model. However, this constraint is much weaker than the constraint on $r_{h}$ as the invisible decay of the second Higgs particle can be very large.

\section{LEP constraint and the electroweak precision test}

The LEP data impose constraints on the ratio of Higgs- $Z-Z$ coupling strength with respect of the SM value $\xi_{\mathcal{H}}^{2}=\left(g_{\mathcal{H} Z Z} / g_{\mathcal{H} Z Z}^{S M}\right)^{2}$ with $\mathcal{H}=h, H$, as shown in figure 10(a) in ref. [82]. In this model, the Higgs- $Z-Z$ coupling strength is suppressed by the mixing between the two Higgs particles

$$
\xi_{h}^{2}=\cos ^{2} \alpha, \quad \xi_{H}^{2}=\sin ^{2} \alpha
$$

The allowed region in the $m_{H}-\sin \alpha$ plane under the constraint from LEP data at $95 \%$ CL is shown in figure 8. This constraint sets an upper bound on the mixing angle in the region with $m_{H}<114 \mathrm{GeV}$, which is however much weaker compared with that from the LHC and the LUX experiments, as can be seen in the figure.

The second Higgs particle in this model gives extra contributions to the gauge boson self-energy diagrams compared with the SM case, which can affect the oblique parameters $S$, $T$ and $U[83,84]$. The shifts of the oblique parameters from the SM values $\Delta X \equiv X-X^{S M}$ 
are given by $[40,85]$

$$
\begin{aligned}
\Delta T= & \frac{3}{16 \pi s_{W}^{2}}\left[\cos ^{2} \alpha\left\{f_{T}\left(\frac{m_{h}^{2}}{m_{W}^{2}}\right)-\frac{1}{c_{W}^{2}} f_{T}\left(\frac{m_{h}^{2}}{m_{Z}^{2}}\right)\right\}\right. \\
& +\sin ^{2} \alpha\left\{f_{T}\left(\frac{m_{H}^{2}}{m_{W}^{2}}\right)-\frac{1}{c_{W}^{2}} f_{T}\left(\frac{m_{H}^{2}}{m_{Z}^{2}}\right)\right\} \\
& \left.-\left\{f_{T}\left(\frac{m_{h}^{2}}{m_{W}^{2}}\right)-\frac{1}{c_{W}^{2}} f_{T}\left(\frac{m_{h}^{2}}{m_{Z}^{2}}\right)\right\}\right], \\
\Delta S= & \frac{1}{2 \pi}\left[\cos ^{2} \alpha f_{S}\left(\frac{m_{h}^{2}}{m_{Z}^{2}}\right)+\sin ^{2} \alpha f_{S}\left(\frac{m_{H}^{2}}{m_{Z}^{2}}\right)-f_{S}\left(\frac{m_{h}^{2}}{m_{Z}^{2}}\right)\right], \\
\Delta U= & \frac{1}{2 \pi}\left[\cos ^{2} \alpha f_{S}\left(\frac{m_{h}^{2}}{m_{W}^{2}}\right)+\sin ^{2} \alpha f_{S}\left(\frac{m_{H}^{2}}{m_{W}^{2}}\right)-f_{S}\left(\frac{m_{h}^{2}}{m_{W}^{2}}\right)\right]-\Delta S,
\end{aligned}
$$

where $m_{W(Z)}$ is the masses of the $W(Z)$ gauge boson, $c_{W}^{2}=m_{W}^{2} / m_{Z}^{2}$ and $s_{W}^{2}=1-c_{W}^{2}$. The functions $f_{T}(x)$ and $f_{S}(x)$ are defined as

$$
\begin{aligned}
& f_{T}(x)= \frac{x \log x}{x-1} \\
& f_{S}(x)=\left\{\begin{array}{l}
\frac{1}{12}\left\{-2 x^{2}+9 x+\left[x^{2}-(6 x-18) / x-1+18\right] x \log x\right. \\
+2 \sqrt{(x-4)}\left(x^{2}-4 x+12\right) \\
\left.\times\left[\tanh ^{-1} \sqrt{x} / \sqrt{x-4}-\tanh ^{-1}(x-2) / \sqrt{(x-4) x}\right]\right\}, \quad \text { for } 0<x<4 \\
\frac{1}{12}\left\{-2 x^{2}+9 x+\left[x^{2}-6 x-18 /(x-1)+18\right] x \log x\right. \\
\left.+\sqrt{(x-4)}\left(x^{2}-4 x+12\right) \log \frac{1}{2}(x-\sqrt{(x-4) x}-2)\right\}, \text { for } x>4 .
\end{array}\right.
\end{aligned}
$$

The constraints from the oblique parameters given in ref. $[85,86]$ can be translated into constraints on the mass of the second Higgs particle and the mixing angle. We show the $95 \%$ CL allowed region in the $m_{H}-\sin \alpha$ plane in figure 8 . It can be seen that this constraint is weaker compared with the LHC constraint and the LUX constraint.

\section{Combined results}

We combine the constraints from all the above mentioned observables such as the DM relic density, the DM-nucleon scattering cross section, the signal strength of the SM-like Higgs particle, the Higgs- $Z-Z$ coupling strength and the oblique parameters on the parameter space satisfying $\varphi_{c} / T_{c}>1$. About $2 \times 10^{5}$ sample points surviving all the constraints are obtained. The frequency distributions of the 6 free parameters after considering the phenomenological constraints are shown in figure 4 .

The allowed region in the $m_{H}-\sin \alpha$ plane is shown in figure 8 . As shown in the figure, the most stringent constraints come from the data of the LHC and the LUX experiments. It can be seen that in the region where the mass of the second Higgs particle is nearly degenerate with that of the SM-like Higgs particle, the LUX constraint is significantly relaxed due to the destructive interference between the contributions from the two Higgs particles. Consequently, in this region the upper bound on the mixing angle is set by the 


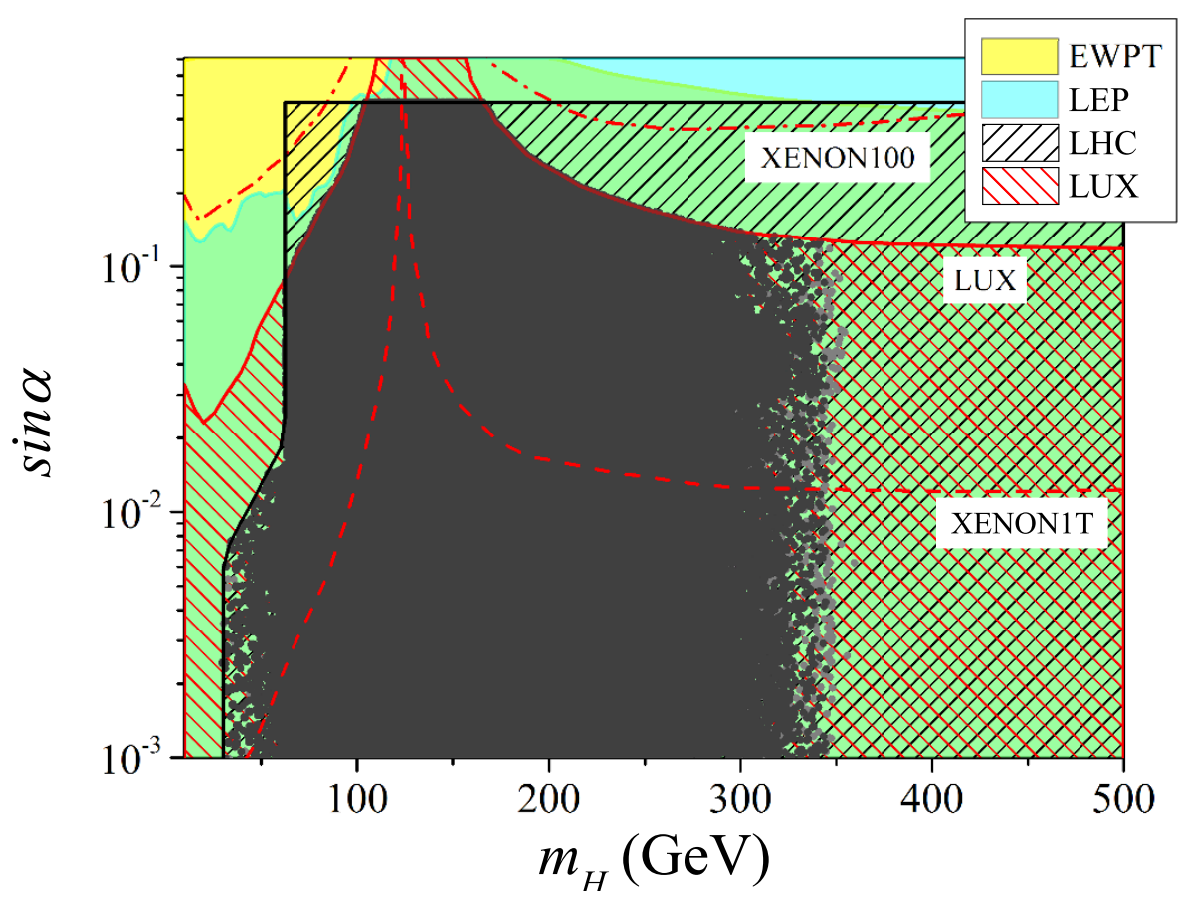

Figure 8. Allowed region in the $m_{H}-\sin \alpha$ plane satisfying $\varphi_{c} / T_{c}>1$ and all the constraints from the electroweak precision test (EWPT) at 95\% CL, the LEP data at 95\% CL, the Higgs search results at LHC, and the upper bound on DM-nucleon scattering cross section from the LUX experiment. The red dot-dashed line is the upper bound on the mixing angle from the $90 \% \mathrm{CL}$ XENON100 constraint and the red dashed line is that from the projected exclusion limit of the future XENON1T experiment. The dots are the sample points satisfying $\varphi_{c} / T_{c}>\mathcal{E}$ and all the constraints with $\mathcal{E}=1.2$ (dark gray) and $\mathcal{E}=1$ (light gray), respectively.

LHC data which leads to $\alpha \lesssim 28^{\circ}$. In the region where $m_{H}<m_{h} / 2$, the mixing angle is further constrained, as the invisible decay of the SM-like Higgs particle is opened. In other regions the upper limit on the mixing angle is determined by the LUX data, for instance, $\alpha \lesssim 5.7^{\circ}$ at $m_{H}=350 \mathrm{GeV}$. As shown by the dots, the requirement of a strongly first order EWPhT sets an upper bound on the mass of the second Higgs particle around $350 \mathrm{GeV}$ for $\mathcal{E}=1$, which is expected as the contributions of very heavy particles to effective potential is suppressed exponentially. As shown by the dark gray dots, when considering $\mathcal{E}=1.2$ the upper bound on the mass of the second Higgs particle becomes lower. But the difference between the upper bound for $\mathcal{E}=1.2$ and that for $\mathcal{E}=1$ is within $10 \mathrm{GeV}$. A lower bound on the mass of the second Higgs particle around $30 \mathrm{GeV}$ is also imposed due to the constraint on $\lambda$ from the LHC data.

The future XENON1T experiment can push the upper bound on $\sigma_{\mathrm{SI}}$ down to $\sim$ $10^{-47} \mathrm{~cm}^{2}$ [79]. The constraint from the projected exclusion limit of the future XENON1T experiment is also shown in figure 8. It can be seen that a significant proportion of the parameter space can be ruled out by the future XENON1T experiment. The mixing angle can be further constrained to one order of magnitude lower compared with the result of the LUX experiment, for instance $\alpha \lesssim 0.57^{\circ}$ at $m_{H}=350 \mathrm{GeV}$. 


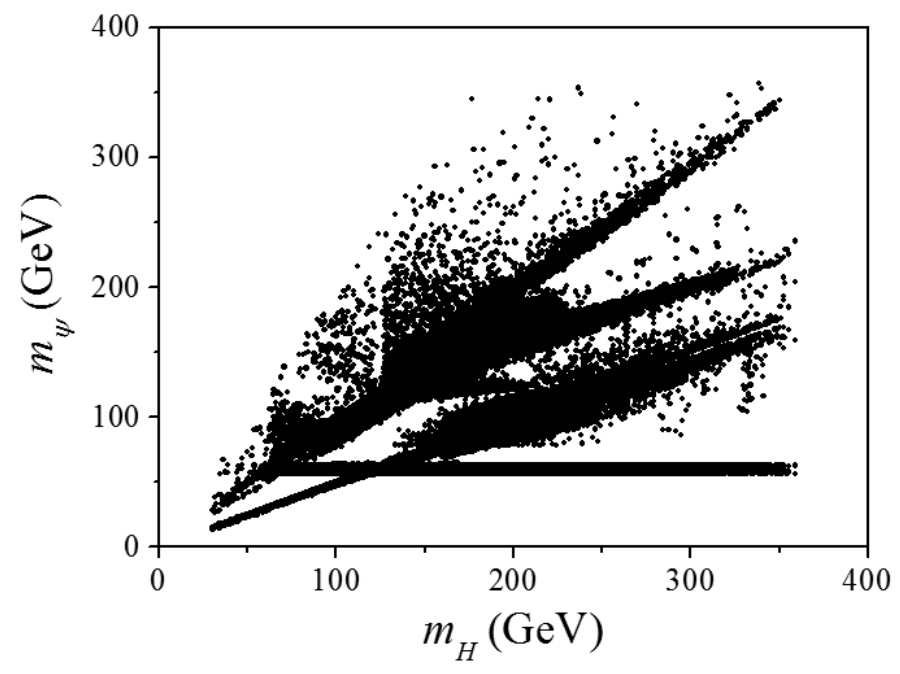

Figure 9. Allowed values of $m_{H}$ and $m_{\psi}$ from the sample points satisfying $\varphi_{c} / T_{c}>1$ and all the phenomenological constraints (see text for detailed explanation).

The allowed values of $m_{H}$ and $m_{\psi}$ from the sample points are shown in figure 9 . The DM particle mass is solved from the DM thermal relic density which leads to a five-fold ambiguity. As shown in the figure, there are three branches which correspond to the two resonant annihilations when $m_{\psi} \approx m_{h, H} / 2$ and the threshold of DM annihilation into Higgs particles. It can be seen that the DM particle mass is predicted to be in the range $\sim 15-350 \mathrm{GeV}$. The distribution of $y_{\psi}$ is also significantly changed by the constraint from DM thermal relic density, as shown in figure 4.

\section{Conclusion}

In summary, we have systematically explored the parameter space of the singlet fermionic DM model which can lead to strongly enough first order EWPhT as required by electroweak baryogenesis. We have taken into account the loop-level barrier by including the high temperature approximation up to the terms quartic in $m / T$, and an analytical approximation of the effective potential which well matches both the high- and low-temperature approximations has been introduced, which allows for reliable calculations in low temperature region. It has been shown that the mixing angle is constrained to $\alpha \lesssim 28^{\circ}$ and the mass of the second Higgs particle is in the range $\sim 30-350 \mathrm{GeV}$. The DM particle mass is predicted to be in the range $\sim 15-350 \mathrm{GeV}$. The future XENON1T detector can rule out a large proportion of the parameter space. The constraint can be relaxed when the mass of the SM-like Higgs particle is degenerate with that of the second Higgs particle. In other regions the mixing angle can be further constrained to one order of magnitude lower compared with the result using the LUX data, for instance $\alpha \lesssim 0.57^{\circ}$ at $m_{H}=350 \mathrm{GeV}$. 


\section{Acknowledgments}

This work is supported in part by the National Basic Research Program of China (973 Program) under Grants No. 2010CB833000; the National Nature Science Foundation of China (NSFC) under Grants No. 10975170, No. 10821504, No. 10905084 and No. 11335012; and the Project of Knowledge Innovation Program (PKIP) of the Chinese Academy of Science.

\section{A Renormalization of the Higgs potential}

The counter-terms to renormalize the potential at zero temperature are given by

$$
V_{\mathrm{CT}}(\varphi, s)=-\frac{\delta \mu_{\phi}^{2}}{2} \varphi^{2}+\frac{\delta \lambda_{\phi}}{4} \varphi^{4}-\frac{\delta \mu_{s}^{2}}{2} s^{2}-\frac{\delta \mu_{3}}{3} s^{3}+\frac{\delta \lambda_{s}}{4} s^{4}+\frac{\delta \mu}{2} \varphi^{2} s+\frac{\delta \lambda}{4} \varphi^{2} s^{2} .
$$

We use the following renormalization conditions

$$
\left.\left(\frac{\partial}{\partial \varphi}, \frac{\partial}{\partial s}, \frac{\partial^{2}}{\partial \varphi^{2}}, \frac{\partial^{2}}{\partial s^{2}}, \frac{\partial^{2}}{\partial s \partial \varphi}\right)\left(V_{1}(\varphi, s)+V_{\mathrm{CT}}(\varphi, s)\right)\right|_{(\varphi, s)=\left(\varphi_{0}, s_{0}\right)}=0
$$

and

$$
\left(\left.\frac{\partial}{\partial s}\right|_{(\varphi, s)=\left(0, s_{\varphi}\right)},\left.\frac{\partial}{\partial v}\right|_{(\varphi, s)=\left(\varphi_{s}, 0\right)}\right)\left(V_{1}(\varphi, s)+V_{\mathrm{CT}}(\varphi, s)\right)=0,
$$

where $s_{\varphi}\left(\varphi_{s}\right)$ is the location of the minimum on the $s(\varphi)$ directions. The conditions in eq. (A.2) keep the locations of tree-level VEVs and the mass of the two Higgs particles unchanged, and that in eq. (A.3) keep the locations of the minima on the $s$ and $\varphi$ direction 
unchanged. The solutions of the renormalization conditions eq. (A.2) and (A.3) are

$$
\begin{aligned}
\delta \mu_{\phi}^{2}= & \frac{\varphi_{s}^{3} V_{1}^{(1,0)}\left(\varphi_{0}, s_{0}\right)+2 \varphi_{0}^{3} V_{1}^{(1,0)}\left(\varphi_{s}, 0\right)-\varphi_{0} \varphi_{s}^{3} V_{1}^{(2,0)}\left(\varphi_{0}, s_{0}\right)}{2 \varphi_{0}^{3} \varphi_{s}} \\
\delta \lambda_{\phi}= & \frac{V_{1}^{(1,0)}\left(\varphi_{0}, s_{0}\right)-\varphi_{0} V_{1}^{(2,0)}\left(\varphi_{0}, s_{0}\right)}{2 \varphi_{0}^{3}} \\
\delta \mu_{s}^{2}= & \frac{1}{2 s_{0}^{2}\left(s_{0}-s_{\varphi}\right)^{2} s_{\varphi}}\left\{s _ { 0 } \left[2 s_{0}^{3} V_{1}^{(0,1)}\left(0, s_{\varphi}\right)+s_{\varphi}^{2}\left(-6 s_{0}+4 s_{\varphi}\right) V_{1}^{(0,1)}\left(\varphi_{0}, s_{0}\right)\right.\right. \\
& \left.+2 s_{\varphi}^{2} s_{0}\left(s_{0}-s_{\varphi}\right) V_{1}^{(0,2)}\left(\varphi_{0}, s_{0}\right)+s_{\varphi}^{2} \varphi_{0}\left(2 s_{0}-s_{\varphi}\right) V_{1}^{(1,1)}\left(\varphi_{0}, s_{0}\right)\right] \\
& \left.-\varphi_{0}^{2} s_{0}^{2} s_{\varphi}^{2}\left(s_{0}-s_{\varphi}\right) \delta \lambda\right\} \\
\delta \mu_{3}= & \frac{1}{2 s_{0}^{3}\left(s_{0}-s_{\varphi}\right)^{2} s_{\varphi}}\left\{-2 s_{0}\left[2 s_{0}^{3} V_{1}^{(0,1)}\left(0, s_{\varphi}\right)+s_{\varphi}\left(-3 s_{0}^{2}+s_{\varphi}^{2}\right) V_{1}^{(0,1)}\left(\varphi_{0}, s_{0}\right)\right.\right. \\
& \left.+s_{\varphi} s_{0}\left(s_{0}^{2}-s_{\varphi}^{2}\right) V_{1}^{(0,2)}\left(\varphi_{0}, s_{0}\right)+s_{\varphi} \varphi_{0} s_{0}^{2} V_{1}^{(1,1)}\left(\varphi_{0}, s_{0}\right)\right] \\
& \left.-\varphi_{0}^{2} s_{0}^{2} s_{\varphi}\left(s_{0}^{2}-s_{\varphi}^{2}\right) \delta \lambda\right\}, \\
\delta \lambda_{s}= & \frac{1}{2 s_{0}^{3}\left(s_{0}-s_{\varphi}\right)^{2} s_{\varphi}}\left\{-s_{0}\left[2 s_{0}^{2} V_{1}^{(0,1)}\left(0, s_{\varphi}\right)+s_{\varphi}\left(-4 s_{0}+2 s_{\varphi}\right) V_{1}^{(0,1)}\left(\varphi_{0}, s_{0}\right)\right.\right. \\
& \left.+2 s_{\varphi} s_{0}\left(s_{0}-s_{\varphi}\right) V_{1}^{(0,2)}\left(\varphi_{0}, s_{0}\right)+s_{\varphi} \varphi_{0} s_{0} V_{1}^{(1,1)}\left(\varphi_{0}, s_{0}\right)\right] \\
& \left.-\varphi_{0}^{2} s_{0}^{2} s_{\varphi}\left(s_{0}-s_{\varphi}\right) \delta \lambda\right\}, \\
\delta \mu= & -\delta \lambda s_{0}, \\
\delta \lambda= & \frac{1}{\varphi_{0}^{3} s_{0} \varphi_{s}}\left[\left(3 \varphi_{0}^{2} \varphi_{s}-\varphi_{s}^{3}\right) V_{1}^{(1,0)}\left(\varphi_{0}, s_{0}\right)-2 \varphi_{0}^{3} V_{1}^{(1,0)}\left(\varphi_{s}, 0\right)\right. \\
& \left.-\varphi_{0}^{2} s_{0} \varphi_{s} V_{1}^{(1,1)}\left(\varphi_{0}, s_{0}\right)-\varphi_{0} \varphi_{s}\left(\varphi_{0}^{2}-\varphi_{s}^{2}\right) V_{1}^{(2,0)}\left(\varphi_{0}, s_{0}\right)\right] \\
& \\
&
\end{aligned}
$$

where

$$
V^{(m, n)}(\varphi, s)=\frac{\partial^{(m+n)} V(\varphi, s)}{\partial h^{m} \partial s^{m}} .
$$

\section{B Cross sections for DM annihilation}

The cross sections for DM particles annihilating into the SM fermions and gauge bosons are given by [37]

$$
\begin{aligned}
& \sigma v_{\text {rel }}\left(\bar{\psi} \psi \rightarrow \bar{f} f, W^{+} W^{-}, Z Z\right)=\frac{\left(y_{\psi} \sin \alpha \cos \alpha\right)^{2}}{16 \pi}\left(1-\frac{4 m_{\psi}^{2}}{\mathfrak{s}}\right) \\
& \times\left|\frac{1}{\mathfrak{s}-m_{h}^{2}+i m_{h} \Gamma_{h}}+\frac{1}{\mathfrak{s}-m_{H}^{2}+i m_{H} \Gamma_{H}}\right|^{2} \cdot A_{f, W, Z},
\end{aligned}
$$

where $\Gamma_{h}\left(\Gamma_{H}\right)$ is the total decay width of the SM-like Higgs particle (the second Higgs particle), $\sqrt{\mathfrak{s}}$ denotes the center-of-mass energy, and $A_{f, W, Z}$ stands for the contributions 
from channels with final states $\bar{f} f, W^{+} W^{-}$and $Z Z$

$$
\begin{aligned}
& A_{f}=6 \mathfrak{s}\left(\frac{m_{f}}{\varphi_{0}}\right)^{2} \times\left(1-\frac{4 m_{f}^{2}}{\mathfrak{s}}\right)^{3 / 2}, \\
& A_{W}=4\left(\frac{m_{W}^{2}}{\varphi_{0}}\right)^{2} \times\left(2+\frac{\left(\mathfrak{s}-2 m_{W}^{2}\right)^{2}}{4 m_{W}^{4}}\right) \times \sqrt{1-\frac{4 m_{W, Z}^{2}}{\mathfrak{s}}} .
\end{aligned}
$$

$A_{Z}$ is defined analogously with $A_{W}$ and there is an additional factor of $1 / 2$ for $A_{Z}$.

The cross sections for DM particles annihilating into two identical Higgs particles through $s$-channele are given by [87]

$$
\sigma v_{\text {rel }}^{(\mathrm{s})}(\bar{\psi} \psi \rightarrow \mathcal{H} \mathcal{H})=\frac{1}{2} \kappa_{\mathcal{H}}\left(\mathfrak{s}-4 m_{\psi}^{2}\right)\left|\frac{y_{h} \lambda_{h \mathcal{H} H}}{\mathfrak{s}-m_{h}^{2}+i m_{h} \Gamma_{h}}+\frac{y_{H} \lambda_{H \mathcal{H}}}{\mathfrak{s}-m_{H}^{2}+i m_{H} \Gamma_{H}}\right|^{2},
$$

where $\mathcal{H}$ stands for $H$ or $h$, and $\kappa_{\mathcal{H}}$ is defined as

$$
\kappa_{\mathcal{H}}=\frac{1}{16 \pi \mathfrak{s}^{2}} \sqrt{\mathfrak{s}^{2}-4 \mathfrak{s} m_{\mathcal{H}}^{2}}
$$

The cross sections for DM particles annihilating into two identical Higgs particles through $t$ - and $u$-channel are given by

$$
\begin{aligned}
\sigma v_{\text {rel }}^{(\mathrm{t}+\mathrm{u})}(\bar{\psi} \psi \rightarrow \mathcal{H \mathcal { H }})= & \kappa_{\mathcal{H}} y_{\mathcal{H}}^{4}\left\{\frac{\left(4 m_{\psi}^{2}-m_{\mathcal{H}}^{2}\right)^{2}}{D^{2}-A^{2}}-\log \left|\frac{A+D}{A-D}\right|\left[\frac{\left(\mathfrak{s}+8 m_{\psi}^{2}-2 m_{\mathcal{H}}^{2}\right)}{2 D}\right.\right. \\
& \left.\left.+\frac{\left(16 m_{\psi}^{4}-4 m_{\psi}^{2} \mathfrak{s}-m_{\mathcal{H}}^{4}\right)}{A D}\right]-2\right\},
\end{aligned}
$$

where $A$ and $D$ are defined as

$$
A=\frac{1}{2}\left(2 m_{\mathcal{H}}^{2}-\mathfrak{s}\right), \quad D=\frac{\mathfrak{s}}{2} \beta_{\psi} \beta_{\mathcal{H}},
$$

with $\beta_{\psi}=\sqrt{1-4 m_{\psi}^{2} / \mathfrak{s}}$ and $\beta_{\mathcal{H}}=\sqrt{1-4 m_{\mathcal{H}}^{2} / \mathfrak{s}}$. The interference terms between the $s$ and $u$-, $t$-channels are given by

$$
\begin{aligned}
\sigma v_{\mathrm{rel}}^{(\mathrm{int})}(\bar{\psi} \psi \rightarrow \mathcal{H} \mathcal{H})= & 2 \kappa_{\mathcal{H}} y_{\mathcal{H}}^{2} m_{\psi}\left[\frac{y_{h} \lambda_{h \mathcal{H}}\left(\mathfrak{s}-m_{h}^{2}\right)}{\left(\mathfrak{s}-m_{h}^{2}\right)^{2}+m_{h}^{2} \Gamma_{h}^{2}}+\frac{y_{H} \lambda_{H \mathcal{H}}\left(\mathfrak{s}-m_{H}^{2}\right)}{\left(\mathfrak{s}-m_{H}^{2}\right)^{2}+m_{H}^{2} \Gamma_{H}^{2}}\right] \\
& \times \log \left|\frac{A+D}{A-D}\right|\left(\frac{A}{D}+\frac{1}{2} \frac{\beta_{\psi}}{\beta_{\mathcal{H}}}-2\right) .
\end{aligned}
$$

The cross sections for DM particles annihilating into $h$ and $H$ through $s$-channel are given by

$$
\sigma v_{\mathrm{rel}}^{(\mathrm{s})}(\bar{\psi} \psi \rightarrow h H)=\kappa_{h H}\left(\mathfrak{s}-4 m_{\psi}^{2}\right)\left|\frac{y_{h} \lambda_{H h h}}{\mathfrak{s}-m_{h}^{2}+i m_{h} \Gamma_{h}}+\frac{y_{H} \lambda_{h H H}}{\mathfrak{s}-m_{H}^{2}+i m_{H} \Gamma_{H}}\right|^{2},
$$


where $\kappa_{h H}$ is defined as

$$
\kappa_{h H}=\frac{1}{16 \pi \mathfrak{s}^{2}} \sqrt{\mathfrak{s}^{2}-2 \mathfrak{s}\left(m_{h}^{2}+m_{H}^{2}\right)+\left(m_{h}^{2}-m_{H}^{2}\right)^{2}} .
$$

The cross sections for DM particle annihilation into $h$ and $H$ through $t$ - and $u$-channel are given by

$$
\begin{aligned}
\sigma v_{\mathrm{rel}}^{\mathrm{(t+ \textrm {u } )}}(\bar{\psi} \psi \rightarrow h H)= & 2 \kappa_{h H} y_{h}^{2} y_{H}^{2}\left\{\frac{\left(4 m_{\psi}^{2}-m_{h}^{2}\right)\left(4 m_{\psi}^{2}-m_{H}^{2}\right)}{D^{2}-A^{2}}\right. \\
& -\log \left|\frac{A+D}{A-D}\right|\left[\frac{\left(\mathfrak{s}+8 m_{\psi}^{2}-m_{h}^{2}-m_{H}^{2}\right)}{2 D}\right. \\
& \left.\left.+\frac{\left(16 m_{\psi}^{4}-4 m_{\psi}^{2} \mathfrak{s}-m_{h}^{2} m_{H}^{2}\right)}{A D}\right]-2\right\},
\end{aligned}
$$

where $A$ and $D$ are defined as

$$
A=\frac{1}{2}\left(m_{h}^{2}+m_{H}^{2}-\mathfrak{s}\right), \quad D=\frac{\mathfrak{s}}{2} \beta_{\psi} \beta_{h H},
$$

with

$$
\beta_{h H}=\sqrt{1-\frac{\left(m_{h}+m_{H}\right)^{2}}{\mathfrak{s}}} \sqrt{1-\frac{\left(m_{h}-m_{H}\right)^{2}}{\mathfrak{s}}} .
$$

The interference terms between the $s$ - and $u$-, $t$-channels are given by

$$
\begin{aligned}
\sigma v_{\text {rel }}^{(\mathrm{int})}(\bar{\psi} \psi \rightarrow h H)= & 4 \kappa_{h H} y_{h} y_{H} m_{\psi}\left[\frac{y_{h} \lambda_{H h h}\left(\mathfrak{s}-m_{h}^{2}\right)}{\left(\mathfrak{s}-m_{h}^{2}\right)^{2}+m_{h}^{2} \Gamma_{h}^{2}}+\frac{y_{H} \lambda_{h H H}\left(\mathfrak{s}-m_{H}^{2}\right)}{\left(\mathfrak{s}-m_{H}^{2}\right)^{2}+m_{H}^{2} \Gamma_{H}^{2}}\right] \\
& \times \log \left|\frac{A+D}{A-D}\right|\left(\frac{A}{D}+\frac{1}{2} \frac{\beta_{\psi}}{\beta_{h H}}-2\right) .
\end{aligned}
$$

The physical couplings in this model are given by

$$
\begin{aligned}
y_{\mathcal{H}}= & \left\{\begin{array}{l}
y_{\psi} \sin \alpha, \text { if } \mathcal{H}=h ; \\
y_{\psi} \cos \alpha, \text { if } \mathcal{H}=H .
\end{array}\right. \\
\lambda_{h h h}= & c_{\alpha}^{3} \lambda_{\phi} \varphi_{0}-\frac{1}{2} c_{\alpha}^{2} s_{\alpha} \lambda s_{0}-\frac{1}{2} c_{\alpha}^{2} s_{\alpha} \mu+\frac{1}{2} c_{\alpha} s_{\alpha}^{2} \lambda \varphi_{0}-s_{\alpha}^{3} \lambda_{s} s_{0}+\frac{1}{3} s_{\alpha}^{3} \mu_{3}, \\
\lambda_{h H H}= & c_{\alpha}^{2} s_{\alpha} \lambda s_{0}-\frac{1}{2} s_{\alpha}^{3} \lambda s_{0}+\frac{1}{2} c_{\alpha}^{3} \lambda \varphi_{0}-c_{\alpha} s_{\alpha}^{2} \lambda \varphi_{0}-3 c_{\alpha}^{2} s_{\alpha} \lambda_{s} s_{0} \\
& +3 c_{\alpha} s_{\alpha}^{2} \lambda_{\phi} \varphi_{0}+c_{\alpha}^{2} s_{\alpha} \mu-\frac{1}{2} s_{\alpha}^{3} \mu+c_{\alpha}^{2} s_{\alpha} \mu_{3}, \\
\lambda_{H h h}= & \frac{1}{2} c_{\alpha}^{3} \lambda s_{0}+\frac{1}{2} c_{\alpha}^{3} \mu-c_{\alpha}^{2} s_{\alpha} \lambda \varphi_{0}+3 c_{\alpha}^{2} s_{\alpha} \lambda_{\phi} \varphi_{0}-c_{\alpha} s_{\alpha}^{2} \lambda s_{0} \\
& +3 c_{\alpha} s_{\alpha}^{2} \lambda_{s} s_{0}-c_{\alpha} s_{\alpha}^{2} \mu-c_{\alpha} s_{\alpha}^{2} \mu_{3}+\frac{1}{2} s_{\alpha}^{3} \lambda \varphi_{0}, \\
\lambda_{H H H}= & c_{\alpha}^{3} \lambda_{s} s_{0}-\frac{1}{3} c_{\alpha}^{3} \mu_{3}+\frac{1}{2} c_{\alpha}^{2} s_{\alpha} \lambda \varphi_{0}+\frac{1}{2} c_{\alpha} s_{\alpha}^{2} \lambda s_{0}+\frac{1}{2} c_{\alpha} s_{\alpha}^{2}+s_{\alpha}^{3} \lambda_{\phi} \varphi_{0} .
\end{aligned}
$$

where $c_{\alpha}$ and $s_{\alpha}$ stand for $\cos \alpha$ and $\sin \alpha$, respectively. 


\section{Sphaleron solution with magnetic moment}

The Lagrangian of the gauge and Higgs sectors of the singlet fermionic DM model is given by

$$
\mathcal{L}=-\frac{1}{4} F_{\mu \nu}^{a} F^{a, \mu \nu}-\frac{1}{4} f_{\mu \nu} f^{\mu \nu}+\left(D_{\mu} \Phi\right)^{\dagger}\left(D^{\mu} \Phi\right)+\frac{1}{2} \partial_{\mu} S \partial^{\mu} S-V(\Phi, S, T),
$$

where

$$
\begin{aligned}
F_{\mu \nu}^{a} & =\partial_{\mu} W_{\nu}^{a}-\partial_{\nu} W_{\mu}^{a}+g \epsilon^{a b c} W_{\mu}^{b} W_{\nu}^{c}, \\
f_{\mu \nu} & =\partial_{\mu} a_{\nu}-\partial_{\nu} a_{\mu}, \\
D_{\mu} & ==\partial_{\mu}-\frac{i}{2} g \sigma^{a} W_{\mu}^{a}-\frac{i}{2} g^{\prime} a_{\mu} H,
\end{aligned}
$$

where $W_{\mu}^{a}(a=1,2,3)$ and $a_{\mu}$ are the $\mathrm{SU}(2)_{L}$ and $\mathrm{U}(1)_{Y}$ gauge fields, respectively. The Higgs potential $V(\Phi, S, T)$ is the effective potential at temperature $T$. The corresponding energy functional is given by

$$
E=\int d^{3} x\left[\frac{1}{4} F_{i j}^{a} F_{i j}^{a}+\left(D_{i} \phi\right)^{\dagger}\left(D_{i} \phi\right)+\frac{1}{2} \partial_{i} S \partial_{i} S+V(\Phi, S, T)\right] .
$$

In the limit of vanishing weak mixing angle, $\theta_{w} \approx 0$, the $\mathrm{U}(1)_{Y}$ gauge field decouples, and the sphaleron solution is spherically symmetric. We adopt the ansatz for the fields from refs. [88-91]

$$
\begin{aligned}
g W_{i}^{a} \sigma^{a} d x^{i} & =(1-f(\xi)) F_{a} \sigma^{a}, \\
\Phi & =\frac{\varphi}{\sqrt{2}}\left(\begin{array}{c}
0 \\
h(\xi)
\end{array}\right), \\
S & =s p(\xi),
\end{aligned}
$$

where $\xi \equiv g v r$ is the dimensionless distance, and the functions $F_{a}$ are defined as [89]

$$
\begin{aligned}
& F_{1}=-2 \sin \phi d \theta-\sin 2 \theta \cos \phi d \phi, \\
& F_{2}=-2 \cos \phi d \theta+\sin 2 \theta \sin \phi d \phi, \\
& F_{3}=2 \sin ^{2} \theta d \phi .
\end{aligned}
$$

The sphaleron energy can be minimized by the solving the variational field equations

$$
\begin{aligned}
f^{\prime \prime} & =\frac{2}{\xi^{2}} f(f-1)(1-2 f)+\frac{1}{4} h^{2}(f-1), \\
h^{\prime \prime}+\frac{2}{\xi} h^{\prime} & =\frac{2}{\xi^{2}} h(1-f)^{2}+\frac{1}{g^{2} \varphi^{4}} \frac{\partial V(h, p, T)}{\partial h}, \\
p^{\prime \prime}+\frac{2}{\xi} p^{\prime} & =\frac{1}{g^{2} \varphi^{2} s^{2}} \frac{\partial V(h, p, T)}{\partial p},
\end{aligned}
$$

where the prime denotes the derivative with respect to $\xi$. To ensure the smoothness at the origin and the asymptotic behavior at $\xi \rightarrow \infty$, the boundary conditions for $f(\xi), h(\xi)$ and $p(\xi)$ are given by

$$
f(0)=h(0)=0
$$


and

$$
f(\infty)=h(\infty)=p(\infty)=1 .
$$

Note that the value of $S$ at the origin is not constrained by any condition. The boundary condition for $p(\xi)$ can be obtained from the Taylor expansion of the equations around $\xi=0$, which leads to $p^{\prime}(0)=0$.

For non-vanishing weak mixing angle, $\theta_{W} \neq 0$, the $\mathrm{U}(1)_{Y}$ gauge field must be taken into account because its source term is nonzero. The source term of the $\mathrm{U}(1)_{Y}$ gauge field $a_{i}$ is given by the current

$$
\partial_{i j} f_{i j}=J_{i}=-\frac{i}{2} g^{\prime}\left[\Phi^{\dagger} D_{i} \Phi-\left(D_{i} \Phi\right)^{\dagger} \Phi\right] .
$$

At the leading order in $\theta_{W}, a_{i}$ in the current can be neglected, which leads to

$$
J_{i}=-\frac{1}{2} g^{\prime} \varphi^{2} \frac{1}{r^{2}} h^{2}(\xi)[1-f(\xi)] \epsilon_{3 i j} x_{j} .
$$

Thus, in the presence of a constant background magnetic field $B$ along the $z$-axis, the energy of the $\mathrm{U}(1)_{Y}$ field is given by

$$
E=-\int d^{3} x a_{i}^{\mathrm{bg}} J_{i}
$$

where $a_{i}^{\mathrm{bg}}=-(B / 2) \epsilon_{3 i j} x_{j}$ is the vector potential of the background magnetic field. The sphaleron energy in eq. (C.16) can be rewritten in the form of a magnetic moment $\mu$ along the $z$-axis in the background magnetic field

$$
E=E_{\text {dipole }}=-\mu B
$$

where the magnetic moment $\mu$ is defined as

$$
\mu=\frac{2 \pi}{3} \frac{g^{\prime}}{g^{3} \varphi(T)} \int_{0}^{\infty} d \xi \xi^{2} h^{2}(\xi)[1-f(\xi)] .
$$

Thus, the non-vanishing weak mixing angle gives rise to a sphaleron magnetic moment [88], and the sphaleron solution becomes axially symmetric [92]. In this case, the ansatz for the fields can be chosen as [89]

$$
\begin{aligned}
g^{\prime} a_{i} d x^{i} & =\left[1-f_{0}(\xi)\right] F_{3}, \\
g W_{i}^{a} \sigma^{a} d x^{i} & =[1-f(\xi)]\left(F_{1} \sigma^{1}+F_{2} \sigma^{2}\right)+\left[1-f_{3}(\xi)\right] F_{3} \sigma^{2}, \\
\Phi & =\frac{\varphi}{\sqrt{2}}\left(\begin{array}{c}
0 \\
h(\xi)
\end{array}\right), \\
S & =\operatorname{sp}(\xi),
\end{aligned}
$$

with $i=1,2,3$. The energy functional is

$$
\begin{aligned}
E= & \frac{4 \pi \varphi}{g} \int_{0}^{\infty} d \xi\left\{\frac{8}{3} f^{\prime 2}+\frac{4}{3} f_{3}^{\prime 2}+\frac{8}{\xi^{2}}\left[\frac{2}{3} f_{3}^{2}(1-f)^{2}+\frac{1}{3}\left(f(1-f)+f-f_{3}\right)^{2}\right]\right. \\
& +\frac{4 g^{2}}{3 g^{\prime 2}}\left[f_{0}^{\prime 2}+\frac{2}{\xi^{2}}\left(1-f_{0}\right)^{2}\right]+\frac{1}{2} \xi^{2} h^{\prime 2}+h^{2}\left[\frac{1}{3}\left(f_{0}-f_{3}\right)^{2}+\frac{2}{3}(1-f)^{2}\right] \\
& \left.+\frac{s^{2}}{2 \varphi^{2}} \xi^{2} p^{\prime 2}+\frac{\xi^{2}}{g^{2} \varphi^{4}} V(h, p, T)\right\} .
\end{aligned}
$$


The energy functional can be minimized by solving the variational equations

$$
\begin{aligned}
f^{\prime \prime} & =\frac{2}{\xi^{2}}(f-1)\left[f(f-2)+f_{3}\left(1+f_{3}\right)\right]+\frac{1}{4} h^{2}(f-1), \\
f_{3}^{\prime \prime} & =\frac{2}{\xi^{2}}\left[3 f_{3}+f(f-2)\left(1+2 f_{3}\right)\right]+\frac{1}{4} h^{2}\left(f_{3}-f_{0}\right), \\
f_{0}^{\prime \prime} & =\frac{2}{\xi^{2}}\left(f_{0}-1\right)+\frac{g^{\prime 2}}{4 g^{2}} h^{2}\left(f_{0}-f_{3}\right), \\
h^{\prime \prime}+\frac{2}{\xi} h^{\prime} & =\frac{2}{3 \xi^{2}} h\left[2(1-f)^{2}+\left(f_{0}-f_{3}\right)^{2}\right]+\frac{1}{g^{2} \varphi^{4}} \frac{\partial V(h, p, T)}{\partial h}, \\
p^{\prime \prime}+\frac{2}{\xi} p^{\prime} & =\frac{1}{g^{2} \varphi^{2} s^{2}} \frac{\partial V(h, p, T)}{\partial p},
\end{aligned}
$$

with boundary conditions given by

$$
f(0)=f_{3}(0)=h(0)=0, \quad f_{0}(0)=1, \quad p^{\prime}(0)=0,
$$

and

$$
f(\infty)=f_{3}(\infty)=f_{0}(\infty)=h(\infty)=p(\infty)=1 .
$$

Open Access. This article is distributed under the terms of the Creative Commons Attribution License (CC-BY 4.0), which permits any use, distribution and reproduction in any medium, provided the original author(s) and source are credited.

\section{References}

[1] A.G. Cohen, D.B. Kaplan and A.E. Nelson, Progress in electroweak baryogenesis, Ann. Rev. Nucl. Part. Sci. 43 (1993) 27 [hep-ph/9302210] [InSPIRE].

[2] V.A. Rubakov and M.E. Shaposhnikov, Electroweak baryon number nonconservation in the early universe and in high-energy collisions, Usp. Fiz. Nauk 166 (1996) 493 [hep-ph/9603208] [INSPIRE].

[3] M. Trodden, Electroweak baryogenesis, Rev. Mod. Phys. 71 (1999) 1463 [hep-ph/9803479] [INSPIRE].

[4] M. Quirós, Finite temperature field theory and phase transitions, hep-ph/9901312 [INSPIRE].

[5] A.D. Sakharov, Violation of CP Invariance, c Asymmetry and Baryon Asymmetry of the Universe, Pisma Zh. Eksp. Teor. Fiz. 5 (1967) 32 [InSPIRE].

[6] V.A. Kuzmin, V.A. Rubakov and M.E. Shaposhnikov, On the Anomalous Electroweak Baryon Number Nonconservation in the Early Universe, Phys. Lett. B 155 (1985) 36 [INSPIRE].

[7] A. De Simone, G. Nardini, M. Quirós and A. Riotto, Magnetic Fields at First Order Phase Transition: A Threat to Electroweak Baryogenesis, JCAP 10 (2011) 030 [arXiv:1107.4317] [INSPIRE].

[8] M.E. Shaposhnikov, Baryon Asymmetry of the Universe in Standard Electroweak Theory, Nucl. Phys. B 287 (1987) 757 [InSPIRE].

[9] M.E. Shaposhnikov, Possible Appearance of the Baryon Asymmetry of the Universe in an Electroweak Theory, JETP Lett. 44 (1986) 465 [INSPIRE]. 
[10] M.E. Carrington, The Effective potential at finite temperature in the Standard Model, Phys. Rev. D 45 (1992) 2933 [inSPIRE].

[11] G.W. Anderson and L.J. Hall, The Electroweak phase transition and baryogenesis, Phys. Rev. D 45 (1992) 2685 [inSPIRE].

[12] P.B. Arnold, Phase transition temperatures at next-to-leading order, Phys. Rev. D 46 (1992) 2628 [hep-ph/9204228] [INSPIRE].

[13] P.B. Arnold and O. Espinosa, The Effective potential and first order phase transitions: Beyond leading-order, Phys. Rev. D 47 (1993) 3546 [Erratum ibid. D 50 (1994) 6662] [hep-ph/9212235] [INSPIRE].

[14] M. Dine, R.G. Leigh, P.Y. Huet, A.D. Linde and D.A. Linde, Towards the theory of the electroweak phase transition, Phys. Rev. D 46 (1992) 550 [hep-ph/9203203] [INSPIRE].

[15] CMS collaboration, S. Chatrchyan et al., Observation of a new boson at a mass of $125 \mathrm{GeV}$ with the CMS experiment at the LHC, Phys. Lett. B 716 (2012) 30 [arXiv:1207.7235] [INSPIRE].

[16] ATLAS collaboration, G. Aad et al., Observation of a new particle in the search for the Standard Model Higgs boson with the ATLAS detector at the LHC, Phys. Lett. B 716 (2012) 1 [arXiv:1207.7214] [InSPIRE].

[17] Planck collaboration, P.A.R. Ade et al., Planck 2013 results. XVI. Cosmological parameters, arXiv:1303.5076 [INSPIRE].

[18] V. Silveira and A. Zee, Scalar phantoms, Phys. Lett. B 161 (1985) 136 [inSPIRE].

[19] J. McDonald, Gauge singlet scalars as cold dark matter, Phys. Rev. D 50 (1994) 3637 [hep-ph/0702143] [INSPIRE].

[20] C.P. Burgess, M. Pospelov and T. Veldhuis, The Minimal model of nonbaryonic dark matter: A Singlet scalar, Nucl. Phys. B 619 (2001) 709 [hep-ph/0011335] [INSPIRE].

[21] H. Davoudiasl, R. Kitano, T. Li and H. Murayama, The New minimal standard model, Phys. Lett. B 609 (2005) 117 [hep-ph/0405097] [INSPIRE].

[22] X.-G. He, T. Li, X.-Q. Li, J. Tandean and H.-C. Tsai, The Simplest Dark-Matter Model, CDMS II Results and Higgs Detection at LHC, Phys. Lett. B 688 (2010) 332 [arXiv:0912.4722] [INSPIRE].

[23] M. Gonderinger, Y. Li, H. Patel and M.J. Ramsey-Musolf, Vacuum Stability, Perturbativity and Scalar Singlet Dark Matter, JHEP 01 (2010) 053 [arXiv:0910.3167] [INSPIRE].

[24] A. Bandyopadhyay, S. Chakraborty, A. Ghosal and D. Majumdar, Constraining Scalar Singlet Dark Matter with CDMS, XENON and DAMA and Prediction for Direct Detection Rates, JHEP 11 (2010) 065 [arXiv: 1003.0809] [INSPIRE].

[25] W.-L. Guo and Y.-L. Wu, The Real singlet scalar dark matter model, JHEP 10 (2010) 083 [arXiv: 1006.2518] [INSPIRE].

[26] Y. Mambrini, Higgs searches and singlet scalar dark matter: Combined constraints from XENON 100 and the LHC, Phys. Rev. D 84 (2011) 115017 [arXiv:1108.0671] [INSPIRE].

[27] W.-L. Guo, L.-M. Wang, Y.-L. Wu, Y.-F. Zhou and C. Zhuang, Gauge-singlet dark matter in a left-right symmetric model with spontaneous CP-violation, Phys. Rev. D 79 (2009) 055015 [arXiv:0811.2556] [INSPIRE]. 
[28] W.-L. Guo, Y.-L. Wu and Y.-F. Zhou, Exploration of decaying dark matter in a left-right symmetric model, Phys. Rev. D 81 (2010) 075014 [arXiv:1001.0307] [INSPIRE].

[29] W.-L. Guo, Y.-L. Wu and Y.-F. Zhou, Searching for Dark Matter Signals in the Left-Right Symmetric Gauge Model with CP Symmetry, Phys. Rev. D 82 (2010) 095004 [arXiv: 1008.4479] [INSPIRE].

[30] W.-L. Guo, Y.-L. Wu and Y.-F. Zhou, Dark matter candidates in left-right symmetric models, Int. J. Mod. Phys. D 20 (2011) 1389 [InSPIRE].

[31] J.-Y. Liu, L.-M. Wang, Y.-L. Wu and Y.-F. Zhou, Two Higgs Bi-doublet Model With Spontaneous $P$ and CP-violation and Decoupling Limit to Two Higgs Doublet Model, Phys. Rev. D 86 (2012) 015007 [arXiv:1205.5676] [InSPIRE].

[32] J.M. Cline and K. Kainulainen, Electroweak baryogenesis and dark matter from a singlet Higgs, JCAP 01 (2013) 012 [arXiv:1210.4196] [INSPIRE].

[33] J.M. Cline, K. Kainulainen, P. Scott and C. Weniger, Update on scalar singlet dark matter, Phys. Rev. D 88 (2013) 055025 [arXiv: 1306.4710] [INSPIRE].

[34] T.A. Chowdhury, M. Nemevšek, G. Senjanović and Y. Zhang, Dark Matter as the Trigger of Strong Electroweak Phase Transition, JCAP 02 (2012) 029 [arXiv:1110.5334] [INSPIRE].

[35] D. Borah and J.M. Cline, Inert Doublet Dark Matter with Strong Electroweak Phase Transition, Phys. Rev. D 86 (2012) 055001 [arXiv: 1204.4722] [INSPIRE].

[36] A. Arhrib, Y.-L.S. Tsai, Q. Yuan and T.-C. Yuan, An Updated Analysis of Inert Higgs Doublet Model in light of the Recent Results from LUX, PLANCK, AMS-02 and LHC, arXiv: 1310.0358 [INSPIRE].

[37] Y.G. Kim, K.Y. Lee and S. Shin, Singlet fermionic dark matter, JHEP 05 (2008) 100 [arXiv:0803.2932] [INSPIRE].

[38] K.Y. Lee, Y.G. Kim and S. Shin, Singlet fermionic dark matter as a natural Higgs portal model, arXiv:0809.2745 [INSPIRE].

[39] Y.G. Kim and S. Shin, Singlet Fermionic Dark Matter explains DAMA signal, JHEP 05 (2009) 036 [arXiv:0901.2609] [INSPIRE].

[40] S. Baek, P. Ko and W.-I. Park, Search for the Higgs portal to a singlet fermionic dark matter at the LHC, JHEP 02 (2012) 047 [arXiv: 1112.1847] [INSPIRE].

[41] S. Baek, P. Ko, W.-I. Park and E. Senaha, Vacuum structure and stability of a singlet fermion dark matter model with a singlet scalar messenger, JHEP 11 (2012) 116 [arXiv: 1209.4163] [INSPIRE].

[42] A. Sommerfeld, Über die Beugung und Bremsung der Elektronen, Ann. Physik 403 (1931) 257.

[43] J. Hisano, S. Matsumoto and M.M. Nojiri, Unitarity and higher order corrections in neutralino dark matter annihilation into two photons, Phys. Rev. D 67 (2003) 075014 [hep-ph/0212022] [INSPIRE].

[44] J. Hisano, S. Matsumoto and M.M. Nojiri, Explosive dark matter annihilation, Phys. Rev. Lett. 92 (2004) 031303 [hep-ph/0307216] [INSPIRE].

[45] M. Cirelli, A. Strumia and M. Tamburini, Cosmology and Astrophysics of Minimal Dark Matter, Nucl. Phys. B 787 (2007) 152 [arXiv:0706.4071] [INSPIRE]. 
[46] N. Arkani-Hamed, D.P. Finkbeiner, T.R. Slatyer and N. Weiner, A Theory of Dark Matter, Phys. Rev. D 79 (2009) 015014 [arXiv:0810.0713] [InSPIRE].

[47] M. Pospelov and A. Ritz, Astrophysical Signatures of Secluded Dark Matter, Phys. Lett. B 671 (2009) 391 [arXiv:0810.1502] [InSPIRE].

[48] J.D. March-Russell and S.M. West, WIMPonium and Boost Factors for Indirect Dark Matter Detection, Phys. Lett. B 676 (2009) 133 [arXiv:0812.0559] [INSPIRE].

[49] S. Cassel, Sommerfeld factor for arbitrary partial wave processes, J. Phys. G 37 (2010) 105009 [arXiv:0903.5307] [INSPIRE].

[50] Z.-P. Liu, Y.-L. Wu and Y.-F. Zhou, Sommerfeld enhancements with vector, scalar and pseudoscalar force-carriers, Phys. Rev. D 88 (2013) 096008 [arXiv: 1305.5438] [INSPIRE].

[51] J. Chen and Y.-F. Zhou, The 130 GeV gamma-ray line and Sommerfeld enhancements, JCAP 04 (2013) 017 [arXiv: 1301.5778] [inSPIRE].

[52] PAMELA collaboration, O. Adriani et al., An anomalous positron abundance in cosmic rays with energies 1.5-100 GeV, Nature 458 (2009) 607 [arXiv: 0810.4995] [INSPIRE].

[53] Fermi LAT collaboration, A.A. Abdo et al., Measurement of the Cosmic Ray e+ plus espectrum from $20 \mathrm{GeV}$ to $1 \mathrm{TeV}$ with the Fermi Large Area Telescope,

Phys. Rev. Lett. 102 (2009) 181101 [arXiv:0905.0025] [INSPIRE].

[54] Fermi LAT collaboration, M. Ackermann et al., Measurement of separate cosmic-ray electron and positron spectra with the Fermi Large Area Telescope, Phys. Rev. Lett. 108 (2012) 011103 [arXiv:1109.0521] [INSPIRE].

[55] AMS collaboration, M. Aguilar, First result from the alpha magnetic spectrometer on the international space station: Precision measurement of the positron fraction in primary cosmic rays of 0.5-350 GeV, Phys. Rev. Lett. 110 (2013) 141102.

[56] H.-B. Jin, Y.-L. Wu and Y.-F. Zhou, Implications of the first AMS-02 measurement for dark matter annihilation and decay, JCAP 11 (2013) 026 [arXiv:1304.1997] [INSPIRE].

[57] M. Fairbairn and R. Hogan, Singlet Fermionic Dark Matter and the Electroweak Phase Transition, JHEP 09 (2013) 022 [arXiv: 1305.3452] [INSPIRE].

[58] J.R. Espinosa, T. Konstandin and F. Riva, Strong Electroweak Phase Transitions in the Standard Model with a Singlet, Nucl. Phys. B 854 (2012) 592 [arXiv:1107.5441] [InSPIRE].

[59] D.J.H. Chung, A.J. Long and L.-T. Wang, The 125 GeV Higgs and Electroweak Phase Transition Model Classes, Phys. Rev. D 87 (2013) 023509 [arXiv:1209.1819] [InSPIRE].

[60] J. Choi and R.R. Volkas, Real Higgs singlet and the electroweak phase transition in the Standard Model, Phys. Lett. B 317 (1993) 385 [hep-ph/9308234] [INSPIRE].

[61] S.W. Ham, Y.S. Jeong and S.K. Oh, Electroweak phase transition in an extension of the standard model with a real Higgs singlet, J. Phys. G 31 (2005) 857 [hep-ph/0411352] [INSPIRE].

[62] A. Ahriche, What is the criterion for a strong first order electroweak phase transition in singlet models?, Phys. Rev. D 75 (2007) 083522 [hep-ph/0701192] [INSPIRE].

[63] S. Profumo, M.J. Ramsey-Musolf and G. Shaughnessy, Singlet Higgs phenomenology and the electroweak phase transition, JHEP 08 (2007) 010 [arXiv:0705.2425] [INSPIRE].

[64] J.M. Cline, G. Laporte, H. Yamashita and S. Kraml, Electroweak Phase Transition and LHC Signatures in the Singlet Majoron Model, JHEP 07 (2009) 040 [arXiv:0905.2559] [INSPIRE]. 
[65] W. Huang, J. Shu and Y. Zhang, On the Higgs Fit and Electroweak Phase Transition, JHEP 03 (2013) 164 [arXiv: 1210.0906] [INSPIRE].

[66] LUX collaboration, D.S. Akerib et al., First results from the LUX dark matter experiment at the Sanford Underground Research Facility, Phys. Rev. Lett. 112 (2014) 091303 [arXiv: 1310.8214] [INSPIRE].

[67] XENON100 collaboration, E. Aprile et al., Dark Matter Results from 225 Live Days of XENON100 Data, Phys. Rev. Lett. 109 (2012) 181301 [arXiv:1207.5988] [INSPIRE].

[68] S.R. Coleman and E.J. Weinberg, Radiative Corrections as the Origin of Spontaneous Symmetry Breaking, Phys. Rev. D 7 (1973) 1888 [INSPIRE].

[69] L. Dolan and R. Jackiw, Symmetry Behavior at Finite Temperature, Phys. Rev. D 9 (1974) 3320 [INSPIRE].

[70] D. Comelli, D. Grasso, M. Pietroni and A. Riotto, The Sphaleron in a magnetic field and electroweak baryogenesis, Phys. Lett. B 458 (1999) 304 [hep-ph/9903227] [INSPIRE].

[71] K. Enqvist, Primordial magnetic fields, Int. J. Mod. Phys. D 7 (1998) 331 [astro-ph/9803196] [INSPIRE].

[72] P. Gondolo and G. Gelmini, Cosmic abundances of stable particles: Improved analysis, Nucl. Phys. B 360 (1991) 145 [InSPIRE].

[73] G. Bélanger, F. Boudjema, A. Pukhov and A. Semenov, MicrOMEGAs: A Program for calculating the relic density in the MSSM, Comput. Phys. Commun. 149 (2002) 103 [hep-ph/0112278] [INSPIRE].

[74] G. Bélanger, F. Boudjema, S. Kraml, A. Pukhov and A. Semenov, Relic density of neutralino dark matter in the MSSM with CP-violation, Phys. Rev. D 73 (2006) 115007 [hep-ph/0604150] [INSPIRE].

[75] G. Bélanger, F. Boudjema, A. Pukhov and A. Semenov, MicrOMEGAs_3: A program for calculating dark matter observables, Comput. Phys. Commun. 185 (2014) 960 [arXiv: 1305.0237] [INSPIRE].

[76] H.-Y. Cheng and C.-W. Chiang, Revisiting Scalar and Pseudoscalar Couplings with Nucleons, JHEP 07 (2012) 009 [arXiv:1202.1292] [INSPIRE].

[77] A. Crivellin, M. Hoferichter and M. Procura, Accurate evaluation of hadronic uncertainties in spin-independent WIMP-nucleon scattering: Disentangling two- and three-flavor effects, Phys. Rev. D 89 (2014) 054021 [arXiv: 1312.4951] [InSPIRE].

[78] V. Cirigliano, M.L. Graesser, G. Ovanesyan and I.M. Shoemaker, Shining LUX on Isospin-Violating Dark Matter Beyond Leading Order, arXiv:1311.5886 [INSPIRE].

[79] XENON1T collaboration, E. Aprile, The XENON1T Dark Matter Search Experiment, arXiv:1206. 6288 [INSPIRE].

[80] J. Ellis and T. You, Updated Global Analysis of Higgs Couplings, JHEP 06 (2013) 103 [arXiv: 1303.3879] [INSPIRE].

[81] CMS collaboration, S. Chatrchyan et al., Search for a standard-model-like Higgs boson with a mass in the range 145 to $1000 \mathrm{GeV}$ at the LHC, Eur. Phys. J. C 73 (2013) 2469 [arXiv: 1304.0213] [INSPIRE].

[82] LEP Working Group for Higgs Boson searches, ALEPH, DELPHI, L3, OPAL 
collaboration, R. Barate et al., Search for the standard model Higgs boson at LEP, Phys. Lett. B 565 (2003) 61 [hep-ex/0306033] [INSPIRE].

[83] M.E. Peskin and T. Takeuchi, A New constraint on a strongly interacting Higgs sector, Phys. Rev. Lett. 65 (1990) 964 [InSPIRE].

[84] I. Maksymyk, C.P. Burgess and D. London, Beyond S, T and U, Phys. Rev. D 50 (1994) 529 [hep-ph/9306267] [InSPIRE].

[85] V. Barger, P. Langacker, M. McCaskey, M.J. Ramsey-Musolf and G. Shaughnessy, LHC Phenomenology of an Extended Standard Model with a Real Scalar Singlet, Phys. Rev. D 77 (2008) 035005 [arXiv:0706 .4311] [INSPIRE].

[86] M. Baak et al., Updated Status of the Global Electroweak Fit and Constraints on New Physics, Eur. Phys. J. C 72 (2012) 2003 [arXiv:1107.0975] [INSPIRE].

[87] H.-Y. Qin, W.-Y. Wang, and Z.-H. Xiong, A simple singlet fermionic dark-matter model revisited, Chin. Phys. Lett. 28 (2011) 111202.

[88] F.R. Klinkhamer and N.S. Manton, A Saddle Point Solution in the Weinberg-Salam Theory, Phys. Rev. D 30 (1984) 2212 [INSPIRE].

[89] F.R. Klinkhamer and R. Laterveer, The Sphaleron at finite mixing angle, Z. Phys. C 53 (1992) 247 [INSPIRE].

[90] J. Choi, Sphalerons in the standard model with a real Higgs singlet, Phys. Lett. B 345 (1995) 253 [hep-ph/9409360] [InSPIRE].

[91] K. Enqvist and I. Vilja, Sphalerons in the singlet majoron model, Phys. Lett. B 287 (1992) 119 [INSPIRE].

[92] N.S. Manton, Topology in the Weinberg-Salam Theory, Phys. Rev. D 28 (1983) 2019 [INSPIRE]. 